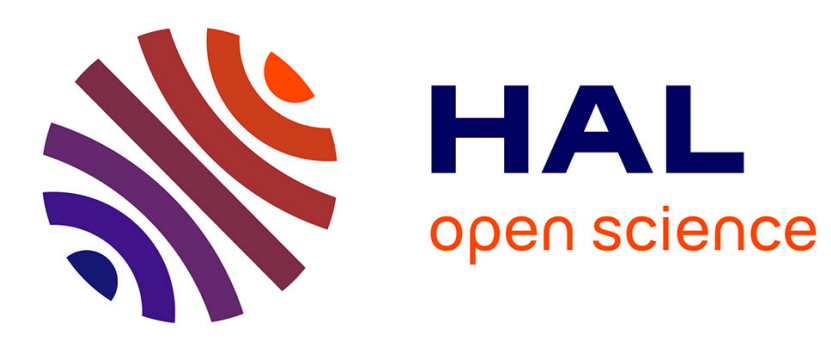

\title{
Arbitrage theory for non convex financial market models
} Emmanuel Lépinette, Tuan Tran Quoc

\section{To cite this version:}

Emmanuel Lépinette, Tuan Tran Quoc. Arbitrage theory for non convex financial market models. 2015. hal-01205876

\author{
HAL Id: hal-01205876 \\ https://hal.science/hal-01205876
}

Preprint submitted on 30 Sep 2015

HAL is a multi-disciplinary open access archive for the deposit and dissemination of scientific research documents, whether they are published or not. The documents may come from teaching and research institutions in France or abroad, or from public or private research centers.
L'archive ouverte pluridisciplinaire HAL, est destinée au dépôt et à la diffusion de documents scientifiques de niveau recherche, publiés ou non, émanant des établissements d'enseignement et de recherche français ou étrangers, des laboratoires publics ou privés. 


\title{
Arbitrage theory for non convex financial market models
}

\author{
Emmanuel LEPINETTE ${ }^{1}$ Tuan TRAN ${ }^{2}$ \\ ${ }^{1}$ Ceremade, UMR CNRS 7534, Dauphine University, Place du Maréchal De Lattre De \\ Tassigny, 75775 Paris cedex 16, France. \\ Email: emmanuel.lepinette@ceremade.dauphine.fr \\ ${ }^{2}$ Dept. of Mathematics and Statistics; McMaster University, \\ Hamilton, ON L8S 4K1, Canada. \\ Email: tuantran@math.mcmaster.ca
}

\begin{abstract}
When dealing with non linear trading costs, e.g. fixed costs, the usual tools from convex analysis are inadequate to characterize an absence of arbitrage opportunity as the mathematical model is no more convex. An unified approach is to describe a financial market model by a liquidation value process. This allows to extend the frictionless models of the classical theory as well as the recent proportional transaction costs models to a large class of financial markets with transaction costs including non linear trading costs. The natural question is to which extent the results of the classical arbitrage theory are still valid when the model is not convex, in particular what does the existence of an equivalent separating probability measure mean ? Our contribution is a first attempt to characterise the absence of arbitrage opportunity in non convex financial market models.
\end{abstract}

Keywords and phrases: Arbitrage theory, Liquidation value, Transaction costs, European options.

2000 MSC: 60G44, G11-G13.

\section{Introduction}

In classical financial market models, the main assumption is the absence of friction, i.e. risky assets can be traded at their instantaneous prices without taking into account transaction costs or liquidity costs as in practice. Arbitrage theory is well developed for such models and one of the main results is the fundamental theorem of asset pricing by Dalang-Morton-Willinger in a discrete-time setting [23, Theorem 2.1.1]. This is a list of equivalent conditions characterising the absence of arbitrage opportunities NA, i.e. $R_{T} \cap L_{+}^{0}=$ $\{0\}$ where $R_{T}$ is the set of all terminal real-valued portfolio processes at time 
$T>0$ starting from a zero initial endowment at time $t=0$ and $L_{+}^{0}$ is the set of all non negative random variables. It is based on the Kreps-Yan theorem which allows to characterise the condition NA as equivalent to the existence of a risk neutral probability measure under which the price process is a martingale. For frictionless continuous-time models, the classical theory was developed by Delbaen and Schachermayer, see [10]. The main result is that there is no arbitrage if and only if there is an equivalent local martingale measure.

Arbitrage theory for financial market models with transaction costs was initiated by Jouini and Kallal [15] for stock markets. Risky assets are modelled by bid-ask intervals $\left[S^{b}, S^{a}\right]$. The bid price $S^{b}$ (resp. the ask price $S^{a}$ ) is the cash (expressed in some numéraire) the agent obtains (resp. pays) when selling (resp. buying) one unit of risky asset. This is a generalisation of frictionless models where it is supposed that $S^{b}=S^{a}=S$ contrarily to what it is observed in practice, e.g. in presence of order books. When $S^{b}<S^{a}$, we may introduce the mid-price $S:=\left(S^{b}+S^{a}\right) / 2$ so that $S^{b}=(1-\epsilon) S$ and $S^{a}=(1+\epsilon) S$ where $\epsilon:=\left(S^{a}-S^{b}\right) /(2 S)$. The coefficient $\epsilon$ may be interpreted as a proportional transaction costs coefficient, see [13], i.e. the agent needs to pay the proportional transaction cost $\epsilon S$ when selling or buying one unit of risky asset at price $S$.

Nowadays, there are many papers on financial market models with proportional transaction costs as introduced by Schachermayer and Kabanov. Their approach has been successfully developed and leads to interesting problems due to the tractability of the model, such as hedging and pricing [7, 14], optimal consumption $[9,30]$. The arbitrage theory for financial market models with proportional transaction costs is a topic of growing interest. One of the main results is the generalisation of the work due to Jouini and Kallal with one risky asset [15], i.e. the equivalence between absence of arbitrage opportunity and the existence of a martingale evolving in the bid-ask spreads [13, 31]. Kabanov provided an unified approach by introducing geometrical financial market models, [23, Chaper 3], where it is possible to exchange any risky asset to another one paying proportional transaction costs. The portfolio positions are vector-valued as they are expressed in physical units contrarily to frictionless models where the liquidation value is a random linear function of the financial position. The important concept in this framework is the socalled solvency cone of all solvent positions, i.e. portfolio positions that can be liquidated without any debt. The usual no arbitrage conditions exclude the possibility of an agent obtaining a solvent terminal portfolio position 
(resp. a non negative liquidation value) when starting from the zero initial endowment except the zero terminal value. Versions of the fundamental theorem of asset pricing was obtained in [19] and [31] for discrete-time models and in [12] for continuous models. Absence of arbitrage opportunity is mainly characterised by the existence of martingales evolving in the positive dual of the solvency cone [23, Theorems 3.2.1 and 3.2.2], see also [21, 20, 22, 24].

In practice, transaction costs may be decomposed into two parts: a brokerage fee and a liquidity cost. Typically, when the trading volume is small, the liquidity cost is negligible while the unit brokerage fee is relatively large compared to the portfolio value. The unit cost tends to gradually decrease as trading volume increases, provided that the trading volume is of moderate size. This can be understood as a kind of order size priority and can be observed at most of security exchanges nowadays. As a result, the brokerage fee is in general a concave function of the trading volume. It is therefore reasonable to study financial market models with concave transaction costs. There is a number of papers in this direction, but they do not study no arbitrage conditions, see for example [27] and [32]. When trading volume is large compared to the total market volume, transactions may be realized at prices far from the best bid-ask prices, due to the lack of supply or demand of securities. Consequently, the liquidity costs appear to be far from being non negligible and should be a convex function of the trading volume. Moreover, not only large transactions make prices executed higher but might also have a significant lasting effect on future prices.

Modelling market impact and liquidity risk has become a very active research subject in mathematical finance. In their seminal paper [1], Almgren and Chris suppose that market impact is the result of temporary and permanent impacts, both depending on the trading intensity. Therefore, risky assets are traded through infinitely small orders so that block trades take time to set up when avoiding substantial, if not infinite, transaction costs. On the other hand, Bank and Baum [3] assume that the price process is continually impacted by the cumulative holdings of the large trader while Cetin, Jarrow and Protter [6] suppose that it is only temporarily impacted by the instantaneous traded volume. In the latter model, the dependence of liquidity cost on trading size is modelled by a stochastic supply curve which represents unit prices depending on trading sizes. This approach allows block trades to be instantaneously executed with finite cost. This is extended in presence of permanent price impact in [4].

To our best knowledge, the main contribution to arbitrage theory for fi- 
nancial market models with fixed costs is due to Jouini, Kallal and Napp [16]. The authors establish the equivalence between an absence of arbitrage opportunity, the no weak arbitrage condition of our paper, and the existence of an absolutely continuous martingale probability measure, at least for a finite probability space. However, they restrict themselves to financial market models with only fixed transaction costs.

In this paper, we consider the general discrete-time model of [25] defined by a liquidation rule which takes into account both transaction and liquidity costs in line with [6]. As we are mainly interested in instantaneous liquidity costs rather than spreading effect of order executions, the liquidation function is assumed to be super-additive, i.e. $L(x+y) \geq L(x)+L(y)$. The intuition is that a smart trader should look for all possible manners in splitting the quantity $z=x+y$ to minimize the trading costs. It is equivalent for him to work with the optimal liquidation rule given by the super-additive liquidation function

$$
\tilde{L}(z)=\sup \left\{L\left(z_{1}\right)+\cdots+L\left(z_{n}\right): \quad z_{1}+\cdots+z_{n}=z\right\} .
$$

Note that by trading at marginal prices of the supply curve with bounded variation strategies, it is shown in [6] that traders may avoid all liquidity costs if the supply curve is smooth enough. In fact, trading at marginal prices amounts to use the optimal liquidation rule which should avoid the discrepancies in hedging prices between discrete and continuous models [11]. Our model is also related to the models with convex liquidation rule [2], [28] but it includes many types of friction as proportional, fixed and concave transaction costs.

The main contribution of our paper is the characterisation of some no arbitrage criteria for a non convex financial market model defined by a super additive liquidation function. We relate the arbitrage opportunities in our model to the arbitrage opportunities appearing in an enlarged market defined as the smallest conical market containing the initial one. Intuitively, this enlarged market is obtained by ruling out any non-linear liquidation costs in the initial market. If the initial market includes both fixed and proportional transaction costs, then the enlarged market should only contain proportional transaction costs. This is motivated by the natural idea that, when an arbitrage opportunity may be realized in one of the two markets, we may sufficiently rescale the associated strategy for the non-linear liquidation costs to be negligible compared with the linear liquidation costs. The 
immediate consequence is that the weak absence of arbitrage opportunity we study is equivalent in the two models and is characterised by the existence of a separating probability measure, i.e. as in the frictionless case.

The paper is organized as follows. In section 2, the model is introduced with basic properties and it is illustrated with several examples. Section 3 is devoted to the existence of an separating probability measure for our model. We show that it is equivalent to an absence of asymptotic arbitrage opportunity as well as the existence of an separating probability measure for the associated enlarged conic model. In Section 4, we study a weak absence of arbitrage opportunity which is well adapted to financial market models with both fixed and proportional transaction costs. We show that this weak no arbitrage condition holds in our model if and only if it also holds in the associated enlarged conic model. The implication is that fixed costs may be considered as negligible as we may sufficiently rescale the trading strategies.

More interesting, we show that the traditional no arbitrage condition (NA) of the frictionless arbitrage theory ${ }^{1}$ holds in the non convex model if and only if the weak no arbitrage condition holds. In particular, under some mild conditions, we finally deduce in the two dimensional case that NA holds if and only if the enlarged conic model satisfies the classical no free lunch arbitrage condition or, equivalently, there is a martingale evolving in the associated bid-ask interval.

At last, we propose to compare the super-hedging price of an European option for the non convex model we consider to the price in the enlarged market, i.e. with only linear costs. The conclusion of our work is that the general non convex financial market model we study is strongly related to the enlarged conic model obtained by neglecting any non-linear liquidation costs, i.e. with only proportional transaction costs.

\section{Notations.}

$e_{1}=(1,0, \cdots, 0) \in \mathbf{R}^{d}, d \geq 1$.

$\mathbb{Q}$ is the set of all rational numbers.

For some subset $G \subseteq \mathbf{R}^{d}, x \geq_{G} y \Leftrightarrow x-y \in G$.

\footnotetext{
${ }^{1}$ The NA condition means that the only non negative portfolio terminal liquidation value when starting from the zero initial endowment is zero.
} 
$\mathbf{R}_{+}^{d}$ is the set of all vectors in $\mathbf{R}^{d}$ having only non negative components and we set $\mathbf{R}_{++}^{d}=\left(\mathbf{R}_{+} \backslash\{0\}\right)^{d}$.

$\mathbb{E}$ designates the expectation of a random variable. When necessary, we denote it by $\mathbb{E}_{Q}$ if considered under $Q \sim \mathrm{P}$ where $\mathrm{P}$ is a probability measure.

If $E$ is a topological space endowed with its Borel $\sigma$-algebra and $\mathcal{F}$ is a $\sigma$ algebra, $L^{0}(E, \mathcal{F})$ (resp. $\left.-L_{+}^{0}(E, \mathcal{F})\right)$ is the metric space of all $E$-valued random variables (resp. non negative random variables) which are $\mathcal{F}$-measurable. More generally, $L^{p}(E, \mathcal{F}, \mathrm{P}), p \in[1, \infty)$ (resp. $\left.p=\infty\right)$, is the normed space of all $E$-valued random variables which are $\mathcal{F}$-measurable and admitting a moment of order $p$ under the probability $\mathrm{P}$ (resp. bounded).

For any subset $\mathcal{X}$ of $L^{0}(\mathbf{R}, \mathcal{F})$, we denote by $\overline{\mathcal{X}}^{p}$, where $p \in[1, \infty) \cup\{0\}$, the closure of $\mathcal{X}^{p}:=\mathcal{X} \cap L^{p}(\mathbf{R}, \mathcal{F}, \mathrm{P})$ with respect to the $L^{p}$-topology (the topology of convergence in probability if $p=0$ ). If $Q \sim \mathrm{P}$, we denote by $\overline{\mathcal{X}}^{p}(Q)$ the closure under $Q$.

\section{Model and basic properties}

In the sequel, we present the model which is defined by random solvency sets or, equivalently, by a liquidation value process, see [25]. Let us consider a probability space $(\Omega, \mathcal{F}, \mathrm{P})$ and let $\left(\mathcal{F}_{t}\right)_{t=0, \cdots, T}$ be a discrete-time filtration. We suppose that the portfolio processes we consider are expressed in physical units, i.e. the number of assets an agent holds. Moreover, we suppose that the first component of such portfolios corresponds to a cash account. Precisely, we assume without loss of generality that the first asset is a bond $S^{1}=1$ so that the first component of a portfolio position is an amount of cash. The financial market model we consider is defined by a sequence of random set-valued mappings $\left(G_{t}\right)_{t=0, \cdots, T}$ we interpret as solvency sets satisfying the following conditions.

\section{Condition $\mathrm{G}^{0}$ :}

(i) $G_{t}$ is a.s. a closed set of $\mathbf{R}^{d}, 0 \leq t \leq T$,

(ii) $G$ is adapted, i.e. $\left\{(\omega, x): x \in G_{t}(\omega)\right\} \in \mathcal{F}_{t} \times \mathcal{B}\left(\mathbf{R}^{d}\right), 0 \leq t \leq T$,

(iii) $\mathbf{R}_{+}^{d} \subseteq G_{t}$, a.s., $0 \leq t \leq T$,

(iv) $G_{t}+G_{t} \subseteq G_{t}$, a.s., $0 \leq t \leq T$,

(v) $\lambda G_{t} \subseteq G_{t}, \lambda \geq 1$, a.s., $0 \leq t \leq T$. 
The random set $G_{t}$ is interpreted as the set of all solvent portfolio positions at time $t$. Condition (iv) means that an aggregation of several solvent positions is still solvent. This implies that $n G_{t} \subseteq G_{t}$ for all integer $n$ and $t \in[0, T]$. However, Condition (v) cannot be deduced in general from the first ones. For instance, consider a market with two assets: one risk free bond $B_{t}$ and one risky stock $S_{t}$ whose values are strictly positive. Suppose that the minimal amount of stock allowed to trade is one unit, i.e. the liquidation value of a portfolio position $(x, y)$ is $\mathbf{L}_{t}(x, y)=x B_{t}+\lfloor y\rfloor S_{t}$. Then $G_{t}$ is the set of all portfolio positions $(x, y)$ at time $t$ such that $\mathbf{L}_{t}(x, y) \geq 0$. We may verify that $G$ satisfies the conditions $\mathbf{G}^{0}$ except (v). In this paper we suppose that Condition (v) holds for every $\lambda \geq 1$ as we do not restrict ourselves to integer-valued portfolio positions.

Definition 2.1. A self-financing portfolio process $\left(V_{t}\right)_{t=0, \cdots, T}$ is an $\left(\mathcal{F}_{t}\right)_{t=0, \cdots, T^{-}}$ adapted process such that

$$
V_{t-1}-V_{t} \in G_{t}, \text { a.s, } \quad t=0, \cdots, T .
$$

This definition is natural. When the agent changes the last position $V_{t-1}$ into the new one $V_{t}$ at time $t$, he lets aside the position $-\Delta V_{t}:=V_{t-1}-V_{t}$ which is required to be liquidated without any debt. This is possible as soon as $-\Delta V_{t}$ is a solvent position, i.e. $-\Delta V_{t} \in G_{t}, t=0, \cdots, T$. Associated to $G$, we define a liquidation rule $\left(\mathbf{L}_{t}\right)_{t=0, \cdots, T}$ such that $G_{t}=\left\{z \in \mathbf{R}^{d}: \mathbf{L}_{t}(z) \geq 0\right\}$.

Definition 2.2. The liquidation value process associated to the process $G$ is

$$
\mathbf{L}_{t}(z):=\sup \left\{\alpha \in \mathbf{R}: z-\alpha e_{1} \in G_{t}\right\}, \quad t=0, \cdots, T .
$$

Intuitively, $\mathbf{L}_{t}(z)$ is the maximal cash obtained by liquidating the portfolio position $z$ at time $t$. Similarly, we may also define the cost process $\left(\mathbf{C}_{t}\right)_{t=0, \cdots, T}$ as

$$
\mathbf{C}_{t}(z):=\inf \left\{\alpha \in \mathbf{R}: \alpha e_{1}-z \in G_{t}\right\}, \quad z \in \mathbf{R}^{d}, \quad t=0, \cdots, T .
$$

By definition, $\mathbf{C}_{t}(z)$ is the minimal amount of cash that allows to purchase the portfolio position $z$ at time $t$. We may verify that $\mathbf{L}_{t}(z)=-\mathbf{C}_{t}(-z)$. Recall that, if the random set $G$ satisfies Condition $\mathrm{G}^{0}$, then $G$ is fully characterised by $\mathbf{L}$ since $G_{t}=\left\{z \in \mathbf{R}^{d}: \mathbf{L}_{t}(z) \geq 0\right\}$, see [25]. Moreover, $\mathbf{L}_{t}$ is upercontinuous and super-additive, i.e.

$$
\mathbf{L}_{t}(x+y) \geq \mathbf{L}_{t}(x)+\mathbf{L}_{t}(y), x, y \in \mathbf{R}^{d}, \quad t=0, \cdots, T .
$$


These conditions are satisfied by a large class of financial market models with friction such as proportional, fixed and concave costs. For instance, in the presence of fixed costs, executing an aggregated order $x+y$ is cheaper than splitting it into two orders $x, y$ and then making them executed separately as we avoid the payment of the fixed costs once again.

In presence of liquidation costs, this also holds provided that the optimal liquidation rule is assumed. An example is where the orders are executed at the best price in the queue of an order book which, by definition, takes into account the impact of orders on prices. Notice that the agent's execution ask price is an execution bid price of the counterpart. Then, we should require that for all $y_{1}, y_{2} \in \mathbf{R}_{+}^{d-1}, \mathbf{C}\left(0, y_{1}+y_{2}\right) \leq \mathbf{C}\left(0, y_{1}\right)+\mathbf{C}\left(0, y_{2}\right)$. Indeed, instead of ordering to buy the quantity $y_{1}+y_{2}$ of risky assets, the agent could send two successive orders respectively of $y_{1}$ and $y_{2}$ risky assets. In this case, he takes the risk that other orders in the queue be executed before his second order $y_{2}$ so that the execution bid price's counterpart is higher in the order book than the one he should benefit from by sending only one order $y_{1}+y_{2}$, as there is no room for intermediate orders. In the case where $y_{1}, y_{2} \in-\mathbf{R}_{+}^{d-1}$, this means that the agents sells $\left|y_{1}\right|+\left|y_{2}\right|$ risky assets and obtains the amount of cash $\left|\mathbf{C}\left(0, y_{1}+y_{2}\right)\right|=-\mathbf{C}\left(0, y_{1}+y_{2}\right)$. By a similar argument than the previous one, we deduce that $\left|\mathbf{C}\left(0, y_{1}+y_{2}\right)\right| \geq\left|\mathbf{C}\left(0, y_{1}\right)\right|+\left|\mathbf{C}\left(0, y_{2}\right)\right|$ since the execution of only one order avoids the insertion of other orders making the ask price's counterpart decreasing. Therefore, we also have the inequality $\mathbf{C}\left(0, y_{1}+y_{2}\right) \leq \mathbf{C}\left(0, y_{1}\right)+\mathbf{C}\left(0, y_{2}\right)$. Otherwise, for instance when $y_{1} \in-\mathbf{R}_{-}^{d-1}$, $y_{2} \in \mathbf{R}_{+}^{d-1}$ and $y_{1}+y_{2} \in \mathbf{R}_{+}^{d-1}$, the answer is not so clear. The agent has the choice between sending a unique order $y_{1}+y_{2}$, here a buy order, or simultaneously sending a sell order $y_{1}$ and a buy order $y_{2}$. In the second case, the agent meets respectively the ask prices of the buyer counterpart of the order book and the bid prices of the seller counterpart so that the possibility for another order to insert between the two orders is excluded. The costs are respectively $\mathbf{C}\left(0, y_{1}\right) \leq 0$ and $\mathbf{C}\left(0, y_{2}\right) \geq 0$ so that the agent is inclined to compare the net cost $\mathbf{C}\left(0, y_{1}\right)+\mathbf{C}\left(0, y_{2}\right)$ to the cost $\mathbf{C}\left(0, y_{1}+y_{2}\right)$ of the unique order. This is why it is optimal for him to replace the cost function by

$$
\tilde{\mathbf{C}}(z):=\inf \left\{\mathbf{C}\left(z_{1}\right)+\cdots+\mathbf{C}\left(z_{n}\right): z=z_{1}+\cdots+z_{n}, n \geq 1\right\} .
$$

We then deduce that $\mathbf{L}\left(z_{1}+z_{2}\right) \geq \mathbf{L}\left(z_{1}\right)+\mathbf{L}\left(z_{2}\right)$ as supposed in this paper.

The following result summarises the properties satisfied by $\mathbf{L}$ and confirms the role of the liquidation rule as defined in (2.2). 
Proposition 2.3. Let $G$ be a random set satisfying Condition $G^{0}$ (i)-(iii) and $\mathbf{L}$ be an associated liquidation value process such that:

(a) $\mathbf{L}_{t}$ is $\mathcal{F}_{t} \times \mathcal{B}\left(\mathbf{R}^{d}\right)$-measurable,

(b) $G_{t}=\left\{z \in \mathbf{R}^{d}: \mathbf{L}_{t}(z) \geq 0\right\}$,

(c) $\mathbf{L}_{t}\left(\alpha e_{1}\right)=\alpha, \forall \alpha \in \mathbf{R}$.

Consider the liquidation value $\widetilde{\mathbf{L}}_{t}$ defined by

$$
\widetilde{\mathbf{L}}_{t}(z):=\sup \left\{\mathbf{L}_{t}\left(z_{1}\right)+\cdots+\mathbf{L}_{t}\left(z_{n}\right): z=z_{1}+\cdots+z_{n}, n \geq 1\right\} .
$$

1. Suppose that $\widetilde{\mathbf{L}}_{t}$ is finite and $\widetilde{G}_{t}:=\left\{z: \widetilde{\mathbf{L}}_{t}(z) \geq 0\right\}$ is a closed set. Then, $\widetilde{G}_{t}$ contains $G_{t}$ and satisfies Condition $G^{0}(i)$-(iv). Moreover, $\widetilde{\mathbf{L}}_{t}$ satisfies (2.2) with $\widetilde{G}_{t}$ in place of $G_{t}$.

2. Moreover, if $\mathbf{L}_{t}$ satisfies the additional condition

(d) $\xi-\mathbf{L}_{t}(\xi) e_{1} \in G_{t}, \forall \xi \in L^{0}\left(\mathbf{R}^{d}, \mathcal{F}_{t}\right)$,

then $\widetilde{G}_{t}$ is the smallest set dominating $G_{t}$ that satisfies Condition $G^{0}$ (i)-(iv), i.e.

$$
\widetilde{G}_{t}=\widehat{G_{t}}:=\overline{\left\{\sum z_{k}: z_{k} \in G_{t}\right\}}
$$

The proof of this proposition is given in Appendix. Note that condition (c) means that the first asset is chosen as the numéraire, while condition (d) means that the portfolio after liquidating $\xi$ is $\mathbf{L}_{t}(\xi) e_{1}$, which are natural requirements for a liquidation rule. In the special case where $G$ is convex, $\widetilde{G}$ is conic i.e. models a so-called Kabanov financial market model with proportional transaction costs as shown in the following proposition.

Proposition 2.4. Suppose that the solvency sets $\left(G_{t}\right)_{t=0, \cdots, T}$ are convex and satisfiy conditions $G^{0}$ (i)-(iii). Let $\mathbf{L}$ be an associated liquidation process satisfying the conditions (a)-(b)-(c) of Proposition 2.3. Moreover, assume that $\mathbf{L}_{t}(\cdot)$ is concave a.s. and $\mathbf{L}_{t}(0)=0, t=0, \cdots, T$. Then, the following statements hold:

1. For every $x \in \mathbf{R}^{d}, \widetilde{\mathbf{L}}_{t}(x)$ defined by (2.4) coincides with the right directional derivative of $\mathbf{L}_{t}$ at 0 in the direction $x$, i.e.

$$
\widetilde{\mathbf{L}}_{t}(x)=D_{x+} \mathbf{L}_{t}(0):=\lim _{\lambda \rightarrow 0+} \frac{\mathbf{L}_{t}(\lambda x)}{\lambda}
$$


provided that the limit is finite. The function $\widetilde{\mathbf{L}}_{t}$ is concave, positively homogeneous of degree 1 and satisfies (2.2) with $\widetilde{G}_{t}$ in place of $G_{t}$.

2. The solvency set $\widetilde{G}_{t}:=\left\{z: \widetilde{\mathbf{L}}_{t}(z) \geq 0\right\}$ associated to $\widetilde{\mathbf{L}}_{t}$ is the smallest closed cone containing $G_{t}$, i.e. $\widetilde{G}_{t}=\overline{\mathbf{R}_{+} G_{t}}$.

The proof of this proposition is given in Appendix. The above result is consistent with the model of [6]. Indeed, the authors show that, when the supply curve is smooth enough (i.e. $\mathbf{L}_{t}$ is smooth), by trading at marginal prices ${ }^{2}$, the trader may avoid the liquidation costs, in particular the super hedging price of an European claim is the same with or without liquidation costs, [6]. As shown in the last proposition, trading at marginal prices is exactly trading with the optimal liquidation rule. Moreover, when $\mathbf{L}_{t}$ is smooth around zero, the solvency cones $\left(\widetilde{G}_{t}\right)_{t=0, \cdots, T}$ are half-spaces as $\widetilde{\mathbf{L}}_{t}(\cdot)$ is a linear form. Therefore, the extended market is frictionless.

Remark 2.5. In the definition of $\mathbf{L}$, the numéraire is the first asset. We could also define a liquidation process $\left(\mathbf{L}_{t}^{\zeta}\right)$ associated to some numéraire $\zeta=\left(\zeta_{t}\right)_{t \leq T}$, i.e. a portfolio process such that $\mathbf{L}_{t}^{\zeta}\left(\zeta_{t}\right)=1$ after normalization. Precisely,

$$
\mathbf{L}_{t}^{\zeta}(\xi):=\sup \left\{\alpha \in \mathbf{R}: \xi-\alpha \zeta_{t} \in G_{t}\right\}
$$

In the sequel, we assume that the initial market $\left(G_{t}\right)_{t=0, \cdots, T}$ of interest satisfies Condition $\mathrm{G}^{0}$ and the liquidation function $\mathbf{L}$ is defined by (2.2). We introduce an enlarged market associated to $G$ as follows:

Definition 2.6. Let $G=\left(G_{t}\right)_{t=0, \cdots, T}$ be such that Condition $G^{0}$ holds and let the associated liquidation process $\mathbf{L}=\mathbf{L}^{G}$ be defined by (2.2). The enlarged market is defined by the solvency sets

$$
K_{t}:=\overline{\mathbf{R}_{+} G_{t}}, \quad t=0, \cdots, T .
$$

In the following, $\mathbf{L}^{K}$ denotes the liquidation process defined by (2.2) with $K$ in place of $G$. Observe that, if the market $G$ satisfies Condition $\mathrm{G}^{0}$, then

$$
K_{t}=\overline{\operatorname{conv} G_{t}}=\overline{\operatorname{cone} G_{t}}, \quad t=0, \cdots, T
$$

where conv $G_{t}$ and cone $G_{t}$ respectively designate the convex and the conic hull of $G_{t}$. By definition, the enlarged market $\left(K_{t}\right)_{t \geq 0}$ is the minimal conic

\footnotetext{
2 i.e. trading with infinitesimal amounts of assets at any moment
} 
market that contains $\left(G_{t}\right)_{t \geq 0}$. For instance, if $\left(G_{t}\right)_{t \geq 0}$ models a financial market with both fixed and proportional transaction costs, then $\left(K_{t}\right)_{t \geq 0}$ is the market model with only proportional costs (see the examples below). It is shown in [25, Proposition 2.6 (iv)] that $\mathbf{L}_{t}^{K}$ is the concave hull of $\mathbf{L}_{t}^{G}$ for all $t=1 \cdots T$. More precisely, we have

$$
\mathbf{L}_{t}^{K}(z)=\lim _{\lambda \rightarrow \infty} \frac{\mathbf{L}_{t}^{G}(\lambda z)}{\lambda},
$$

where the function in the right hand side is increasing in $\lambda$. So, when $\mathbf{L}_{t}^{K}(z)$ is finite, we have the decomposition

$$
\mathbf{L}_{t}^{G}(\lambda z)=\lambda \mathbf{L}_{t}^{K}(z)-\lambda \delta_{t}(\lambda, z)
$$

Here, the function $\delta_{t}: \mathbf{R}_{+} \times \mathbf{R}^{d} \rightarrow \mathbf{R}_{+}$is non-increasing in the first argument and satisfies $\lim _{\lambda \rightarrow \infty} \delta(\lambda, z)=0$. Moreover, $\delta_{t}(\lambda, \beta z)=\delta_{t}(\lambda \beta, z)$ for all $\beta \in$ $\mathbf{R}_{+}$. This simple decomposition says that the liquidation value of a portfolio position $z$ consists of two parts: a linear liquidation value part minus a nonlinear costs part. Moreover, by sufficiently rescaling the portfolio position, the non-linear part vanishes. Therefore, the liquidation values of the same large portfolio position in the two markets $G$ and $K$ should be comparable, which will be confirmed when comparing the arbitrage opportunities between the two markets.

Example 2.7 (Financial market model with fixed and proportional transaction costs).

Let us consider a financial market model with two assets: the first one is $a$ bond $B_{t}=1$ for all $t$, and the second one is risky and defined by a bidask interval given by $\left[S^{b}, S^{a}\right]$ where $S^{a}$ and $S^{b}$ are two adapted processes. We suppose that for each transaction such that a non null quantity of risky assets is exchanged, the agent is asked for paying a positive fixed cost defined by a process $\left(c_{t}\right)_{t=0, \cdots, T}$ which is assumed to be bounded. Moreover, we suppose that when the quantity $y$ of risky asset the agent holds is non negative and too small to compensate for the fixed cost, i.e. $y \geq 0$ and $y S_{t}^{b}-c_{t} \leq 0$, then he prefers to give up his risky position. This means that the liquidation value of the position $(x, y)$ is given by

$$
\mathbf{L}_{t}((x, y))=x+\left(y S_{t}^{b}-c_{t}\right)^{+} 1_{y>0}+\left(y S_{t}^{a}-c_{t}\right) 1_{y<0} .
$$


Therefore, the solvency sets of the model are given by $G_{t}:=\left(K_{t}+C_{t}\right) \cup\left[0, C_{t}\right]$ where $C_{t}=c_{t} e_{1}$, while $K \supseteq \mathbf{R}_{+}^{2}$ is the closed proper cone generated by the bid-ask interval $\left[S^{b}, S^{a}\right]$, i.e. the solvency cone in the Kabanov model:

$$
K_{t}=\operatorname{cone}\left(S_{t}^{a} e_{1}-e_{2} ;-S_{t}^{b} e_{1}+e_{2}\right), \quad t=0, \cdots, T .
$$

Observe that, for $\epsilon>0, \epsilon G_{t}$ coincides with the solvency set with the fixed cost $\epsilon c_{t}$ in place of $c_{t}$. Therefore, Condition $\mathbf{G}^{0}$ holds for this model and $K_{t}=\overline{\cup_{\epsilon>0} \epsilon G_{t}}=\overline{\mathbf{R}_{+} G_{t}}$.

Example 2.8 (Financial market with concave transaction costs).

Let us now consider a financial market model with two assets: one riskless asset whose value is constant over time: $B_{t}=1$ for all $t$, and one risky asset whose book values are defined by an adapted process $\left(S_{t}\right)_{t=0, \cdots, T}$. We suppose that, when the trader liquidates a non null quantity $y$ of risky asset, he obtains $y S_{t}-\beta_{t}|y|^{\alpha} S_{t}$, where $\alpha \in[0,1]$ and $\left(\beta_{t}\right)_{t=0, \cdots, T}$ is a non-negative adapted process, i.e. the agent has to pay an extra cost $\beta_{t}|y|^{\alpha} S_{t}$ which is a concave function of $y$. The liquidation function is then given by

$$
\mathbf{L}_{t}((x, y))=x+\left(y-\beta_{t}|y|^{\alpha}\right)^{+} S_{t} 1_{y>0}+\left(y-\beta_{t}|y|^{\alpha}\right) S_{t} 1_{y<0} .
$$

Observe that this model also satisfies Condition $\mathbf{G}^{0}$ except (iv). Note that, if $\alpha=0$, this corresponds to fixed transaction costs and if $\alpha=1$, the transaction costs are proportional. In general, the enlarged market has no friction, i.e. solvency sets are half-spaces. This model is a special example of the concave liquidity model considered in [26].

Example 2.9 (Financial market with piecewise proportional transaction costs).

We still consider a financial market model with two assets: one riskless asset whose value is constant over time: $B_{t}=1$ for all $t$, and one risky asset whose price is defined by an adapted process $\left(S_{t}\right)_{t=0, \cdots, T}$. We suppose that the transaction costs are piecewise proportional to the exchanged volume, i.e.

$$
\mathbf{L}_{t}((x, y))=x+(1-\beta(y, t)) y S_{t}
$$

where $\beta(y, t)$ is a piecewise function, i.e. there exists a sequence $\left(a_{k}\right)_{k=-(n+1), \cdots, k=n+1}$, $n \in \mathbf{N}$, such that

$$
a_{-(n+1)}=-\infty \leq a_{-n} \leq \cdots \leq a_{-1} \leq a_{0}=0 \leq a_{1} \leq \cdots \leq a_{n} \leq a_{n+1}=+\infty
$$


and proportional transaction costs coefficients

$$
\beta_{0}^{-} \leq \cdots \leq \beta_{n}^{-} \leq 0=\beta_{0} \leq \beta_{n}^{+} \leq \cdots \leq \beta_{0}^{+},
$$

such that $\beta=\beta_{k}^{-}$on $\left[a_{-(k+1)}, a_{-k}\right)$ and $\beta=\beta_{k}^{+}$on $\left(a_{k}, a_{k+1}\right], k=0, \cdots, n$. In this model, the agents benefit from lower proportional transaction costs when the traded volume is larger. It is straightforward to check that Condition $\mathbf{G}^{0}$ holds in this model. Moreover, the enlarged market is defined by the cone associated to the bid-ask spreads $\left[S_{t}\left(1-\beta_{n}^{+}\right), S_{t}\left(1-\beta_{n}^{-}\right)\right]$or equivalently by the proportional transaction costs coefficients $\beta_{n}^{+}$and $\beta_{n}^{+}$.

We denote by $\mathrm{R}_{T}^{0}$ the set of all terminal values $V_{T}$ of portfolio processes $V$ such that the initial capital is $V_{-1}=0$, i.e.

$$
\mathrm{R}_{T}^{0}:=\mathrm{R}_{T}^{0}(G):=\sum_{0 \leq t \leq T} L^{0}\left(-G_{t}, \mathcal{F}_{t}\right) .
$$

We also define the associated liquidation values

$$
\mathrm{LV}_{T}^{0}:=\mathrm{LV}_{T}^{0}(G):=\left\{\mathbf{L}_{T}\left(V_{T}\right): V_{T} \in \mathrm{R}_{T}^{0}\right\}
$$

and, more generally, we define $\mathrm{R}_{T}^{u}:=\sum_{u \leq t \leq T} L^{0}\left(-G_{t}, \mathcal{F}_{t}\right)$ and, similarly, $\mathrm{LV}_{T}^{u}$. Observe that $L^{0}\left(-\mathbf{R}_{+}, \mathcal{F}_{T}\right) \subseteq \mathrm{LV}_{T}^{0}$.

The following condition coincides with the no arbitrage condition (NA) of the frictionless arbitrage theory.

Definition 2.10. We say that the financial market model defined by $G$ satisfies the $N A$ condition, i.e. there is no arbitrage opportunity, if the property $\mathrm{LV}_{T}^{0} \cap L^{0}\left(\mathbf{R}_{+}, \mathcal{F}_{T}\right)=\{0\}$ is satisfied.

Let us introduce the convex cone

$$
\mathrm{A}_{T}^{0}:=\left\{\sum_{i=1}^{n} \lambda_{i} X_{i}: \lambda_{i} \geq 0, X_{i} \in \mathrm{LV}_{T}^{0}\right\}
$$

Recall from [25] that

$$
\mathrm{A}_{T}^{0}:=\left\{\lambda X: \lambda \in[0,1], X \in \mathrm{LV}_{T}^{0}\right\}
$$

Therefore, Condition NA is equivalent to $\mathrm{A}_{T}^{0} \cap L^{0}\left(\mathbf{R}_{+}^{d}, \mathcal{F}_{T}\right)=\{0\}$, i.e. the condition NA for the financial market model defined by the enlarged conic set $\mathrm{A}_{T}^{0}$. This is convenient since $\mathrm{A}_{T}^{0}$ is a convex cone, as we shall see in the next section. 


\section{Equivalent separating probability measure (ESPM)}

For classical models without friction, but also for the Schachermayer model [13], portfolio processes are liquidation values of portfolio positions expressed in physical units and absence of arbitrage opportunity is equivalent to the existence of an equivalent separating probability measure (ESPM), i.e. a probability measure $Q \sim \mathrm{P}$ such that $\mathbb{E}_{Q}\left[V_{T}\right] \leq 0$ for all liquidation values $V_{T}$ of terminal portfolio processes starting from the zero initial endowment. Since these models are convex, this allows to formulate a dual characterization of prices super-hedging an European contingent claim. We refer readers to [23, Chapter 2] for a brief review of the literature.

The natural question is whether the existence of such an ESPM also characterizes an absence of arbitrage opportunity in our model. We show in this subsection that the existence of an ESPM for our model is equivalent to the absence of an asymptotic arbitrage opportunity as well as the existence of an ESPM for the convex enlarged market model defined by $\left(K_{t}\right)_{t \leq T}$.

Let us introduce, for any $\mathrm{P}^{\prime} \sim \mathrm{P}$ and $p \in[1, \infty), q \in(1, \infty]$ such that $p^{-1}+q^{-1}=1$, the set

$$
\mathcal{D}^{q}\left(\mathcal{X}, \mathrm{P}^{\prime}\right):=\left\{Q \sim \mathrm{P}^{\prime}: \frac{d Q}{d \mathrm{P}^{\prime}} \in L^{q}\left(\mathbf{R}, \mathcal{F}_{T}, \mathrm{P}^{\prime}\right), \mathbb{E}_{Q} X \leq 0, \forall X \in \mathcal{X}^{p}\right\},
$$

where $\mathcal{X}$ is a convex cone in $L^{0}\left(\mathbf{R}, \mathcal{F}_{T}\right)$ containing $-L^{\infty}\left(\mathbf{R}_{+}, \mathcal{F}_{T}\right)^{3}$. We denote by $\overline{\mathcal{X}}^{0}$ the closure in probability of $\mathcal{X}$ in $L^{0}$ and by $\overline{\mathcal{X}}^{p}, 1 \leq p \leq \infty$, the closure of $\mathcal{X} \cap L^{p}$ for the $L^{p}$ norm topology. For sake of completeness, we provide the proof of the following classical result.

Lemma 3.1. The following conditions are equivalent

(i) $\overline{\mathcal{X}}^{0} \cap L^{0}\left(\mathbf{R}_{+}, \mathcal{F}_{T}, \mathrm{P}\right)=\{0\}$,

(ii) For all $\mathrm{P}^{\prime} \sim \mathrm{P}, \overline{\mathcal{X}}^{p}\left(\mathrm{P}^{\prime}\right) \cap L^{p}\left(\mathbf{R}_{+}, \mathcal{F}_{T}, \mathrm{P}^{\prime}\right)=\{0\}, \quad 1 \leq p<\infty$,

(iii) For all $\mathrm{P}^{\prime} \sim \mathrm{P}, 1 \leq p<\infty, \mathcal{D}^{q}\left(\mathcal{X}, \mathrm{P}^{\prime}\right) \neq \emptyset$.

Proof. The implication $(i) \Rightarrow(i i)$ is straightforward. The implication $(i i) \Rightarrow(i i i)$ is deduced by using the Kreps-Yan theorem based on the HahnBanach separation theorem, [23, Theorem 2.1.4.].

Let us prove the implication $(i i i) \Rightarrow(i)$. Suppose that $(i)$ does not hold and let $\left(\psi_{n}\right)_{n \geq 0}$ be a sequence in $\mathcal{X}$ converging a.s to $\psi \in L^{0}\left(\mathbf{R}_{+}, \mathcal{F}_{T}, \mathrm{P}\right) \backslash\{0\}$.

\footnotetext{
${ }^{3}$ This condition is sufficient for the Kreps-Yan theorem to be valid, [23, Theorem 2.1.4], as we may observe when repeating the proof.
} 
Therefore, $\psi_{n}^{-} \rightarrow 0$ hence $\sup _{n}\left[\psi_{n}\right]^{-}<\infty$, P- a.s. We then choose $\mathrm{P}^{\prime} \sim \mathrm{P}$ such that $\sup _{n}\left[\psi_{n}\right]^{-} \in L^{p}\left(\mathbf{R}_{+}, \mathcal{F}, \mathrm{P}^{\prime}\right)$ and $\psi_{n} \in L^{p}\left(\mathbf{R}, \mathcal{F}, \mathrm{P}^{\prime}\right)$ for all $n$. By assumption, there is $Q \sim \mathrm{P}^{\prime}$ such that $d Q / d \mathrm{P}^{\prime} \in L^{q}\left(\mathbf{R}, \mathcal{F}, \mathrm{P}^{\prime}\right)$ and $\mathbb{E}_{Q} \psi_{n} \leq 0$ for all $n$. Observe that $\sup _{n}\left[\psi_{n}\right]^{-} \in L_{1}(Q)$. Therefore, we may apply the Fatou lemma and deduce that

$$
\mathbb{E}_{Q} \psi \leq \liminf _{n} \mathbb{E}_{Q} \psi_{n} \leq 0
$$

i.e. a contradiction.

Let us denote by ${\overline{\mathrm{A}_{T}^{\infty}}}^{w}$ the closure of $\mathrm{A}_{T}^{\infty}:=\mathrm{A}_{T}^{0} \cap L^{\infty}\left(\mathbf{R}, \mathcal{F}_{T}\right)$ with respect to the weak-star topology $\sigma\left(L^{\infty}(\mathbf{R}, \mathcal{F}, \mathrm{P}), L^{1}(\mathbf{R}, \mathcal{F}, \mathrm{P})\right)$. We introduce the set of equivalent separating probability measures as

$$
\mathcal{D}^{1}(\mathrm{P}):=\left\{Q \sim \mathrm{P}: \frac{d Q}{d \mathrm{P}} \in L^{1}\left(\mathbf{R}_{+}, \mathcal{F}, \mathrm{P}\right), \mathbb{E}_{Q} X \leq 0, \forall X \in \mathrm{LV}_{b, T}^{0}\right\},
$$

where

$$
\mathrm{LV}_{b, T}^{0}:=\left\{X \in \mathrm{LV}_{T}^{0}:\left\|X^{-}\right\|_{\infty}<\infty\right\} .
$$

Applying the lemma above to $\mathcal{X}=\mathrm{A}_{T}^{0}$, we deduce the following theorem that characterizes the existence of an ESPM.

Theorem 3.2. The following conditions are equivalent

(i) ${\overline{\mathrm{A}_{T}^{0}}}^{0} \cap L^{0}\left(\mathbf{R}_{+}, \mathcal{F}_{T}, \mathrm{P}\right)=\{0\}$,

(ii) ${\overline{\mathrm{A}_{T}^{\infty}}}^{w} \cap L^{\infty}\left(\mathbf{R}_{+}, \mathcal{F}_{T}, \mathrm{P}\right)=\{0\}$,

(iii) There exists an ESPM, i.e. $\mathcal{D}^{1}(\mathrm{P}) \neq \emptyset$.

Proof.

(i) $\Rightarrow$ (ii) : Applying Lemma 3.1 with $p=1$, condition (i) implies the existence of $Z \in L^{\infty}\left(\mathbf{R}_{++}, \mathcal{F}_{T}, \mathrm{P}\right)$ such that $\mathbb{E}[Z Y] \leq 0$ for all $Y \in \mathrm{A}_{T}^{1}$. By definition of the weak-star topology, we deduce that $\mathbb{E}[Z Y] \leq 0$ for all $Y \in{\overline{\mathrm{A}_{T}^{\infty}}}^{w}$, hence, $\overline{\mathrm{A}}_{T}^{\infty}{ }^{w} \cap L^{\infty}\left(\mathbf{R}_{+}, \mathcal{F}_{T}\right)=\{0\}$.

(ii) $\Leftrightarrow($ iii $)$ By virtue of the Kreps-Yan theorem [23, Theorem 2.1.4], we get that $(i i)$ is equivalent to $\widetilde{\mathcal{D}}^{1}(P) \neq \emptyset$ where

$$
\widetilde{\mathcal{D}}^{1}(\mathrm{P}):=\left\{Q \sim \mathrm{P}: \frac{d Q}{d \mathrm{P}} \in L^{1}(\mathbf{R}, \mathcal{F}, \mathrm{P}), \mathbb{E}_{Q} X \leq 0, \forall X \in{\overline{\mathrm{A}_{T}^{\infty}}}^{w}\right\} .
$$

We claim that $\widetilde{\mathcal{D}}^{1}(\mathrm{P})=\mathcal{D}^{1}(\mathrm{P})$. Indeed, if $Q \in \widetilde{\mathcal{D}}^{1}(\mathrm{P})$, then $\mathbb{E}_{Q} X \leq 0$ for all $X \in \mathrm{A}_{T}^{\infty}$. Moreover, if $Z \in \mathrm{LV}_{b, T}^{0}$, then $Z \wedge n \in \mathrm{A}_{T}^{\infty}, n \in \mathbf{N}$. Using the 
Fatou lemma, we get that $\mathbb{E}_{Q} Z \leq \liminf _{n} \mathbb{E}_{Q}(Z \wedge n) \leq 0$ which implies that $Q \in \mathcal{D}^{1}(\mathrm{P})$. Reciprocally, consider $Q \in \mathcal{D}^{1}(\mathrm{P})$. By definition, $\mathbb{E}_{Q} X \leq 0$ for all $X \in \operatorname{LV}_{T}^{\infty}$. Since $\mathrm{A}_{T}^{\infty}=\left\{\lambda X, X \in \operatorname{LV}_{T}^{\infty}, \lambda \in[0,1]\right\}$, we deduce that $\mathbb{E}_{Q} X \leq 0$ for all $X \in \mathrm{A}_{T}^{\infty}$. Notice that the mapping $X \mapsto \mathbb{E}_{Q} X$ is a continuous linear mapping with respect to the $\sigma\left(L^{\infty}, L^{1}\right)$-topology. Since the inclusion

$$
\mathrm{A}_{T}^{\infty} \subseteq \mathcal{X}(Q):=\left\{X: \mathbb{E}_{Q} X \leq 0\right\}
$$

holds and $\mathcal{X}(Q)$ is $\sigma\left(L^{\infty}, L^{1}\right)$-closed, this implies that ${\overline{\mathrm{A}_{T}^{\infty}}}^{w} \subseteq \mathcal{X}(Q)$ hence $\mathbb{E}_{Q} X \leq 0$ for all $X \in \overline{\mathrm{A}_{T}^{\infty}}$, i.e. $Q \in \widetilde{\mathcal{D}}^{1}(\mathrm{P})$ and finally $\widetilde{\mathcal{D}}^{1}(\mathrm{P})=\mathcal{D}^{1}(\mathrm{P})$.

$($ iii $) \Rightarrow(i)$ : Recall that, if $\mathrm{P}^{\prime} \sim \mathrm{P}$ and $\mathcal{X}$ is a convex set in $L^{\infty}$, then $\overline{\mathcal{X}}^{w}(\mathrm{P})=\overline{\mathcal{X}}^{w}\left(\mathrm{P}^{\prime}\right)$. Applying this to $\mathcal{X}=\mathrm{A}_{T}^{\infty}$, we then conclude as in the proof showing that $($ iii $) \Rightarrow(i)$ in Lemma 3.1 .

When $G$ is convex, we get that $\mathrm{A}_{T}^{0}=\mathrm{LV}_{T}^{0}$ and we immediately deduce the following result.

Corollary 3.3. Suppose that $G$ is a.s. convex. The following conditions are equivalent:

(i) ${\overline{\mathrm{LV}_{T}^{0}}}^{0} \cap L^{0}\left(\mathbf{R}_{+}, \mathcal{F}_{T}\right)=\{0\}$,

(ii) $\overline{\mathrm{LV}}_{T}^{\infty} \mathrm{w} \cap L^{\infty}\left(\mathbf{R}_{+}, \mathcal{F}_{T}\right)=\{0\}$,

(iii) There is an equivalent separating probability measure, i.e. $\mathcal{D}^{1}(\mathrm{P}) \neq \emptyset$.

The following result implies that the sets of ESPMs coincide for both the initial market $G$ and the enlarged one $K$.

Theorem 3.4. We have the equality ${\overline{\mathrm{A}_{T}^{0}(G)}}^{0}={\overline{\mathrm{A}_{T}^{0}(K)}}^{0}$.

Proof. It is trivial that ${\overline{\mathrm{A}_{T}^{0}(G)}}^{0} \subseteq{\overline{\mathrm{A}_{T}^{0}(K)}}^{0}$. Let us prove the inclusion $\mathrm{A}_{T}^{0}(K) \subseteq{\overline{\mathrm{A}_{T}^{0}(G)}}^{0}$. To do so, consider $\xi \in \mathrm{A}_{T}^{0}(K)$, i.e. $\xi e_{1} \in \mathbf{R} e_{1}$ a.s. and $\xi e_{1}=\sum_{t=0}^{T} \xi_{t}$ where $\xi_{t} \in L^{0}\left(-K_{t}, \mathcal{F}_{t}\right), t \leq T$. Consider

$$
\begin{aligned}
& \zeta^{n}:=\xi e_{1}-(T+1) n^{-1} e_{1} \\
& \zeta_{t}^{n}:=\xi_{t}-n^{-1} e_{1}, \quad t \leq T, \quad n \geq 1 .
\end{aligned}
$$

We have $\zeta^{n} \rightarrow \xi e_{1}$ as $n \rightarrow \infty$.

Let us introduce $K_{t}^{0}:=\cup_{\lambda>0} \lambda G_{t}$, and let us show that $\zeta_{t}^{n} \in L^{0}\left(-K_{t}^{0}, \mathcal{F}_{t}\right)$ for all $t \leq T$. As $K_{t}$ is a convex cone and $e_{1} \in \operatorname{int} K_{t}, K_{t}+n^{-1} e_{1} \subseteq \operatorname{int} K_{t}$, by [29, Theorem 6.1]. Moreover, since $K_{t}=\overline{K_{t}^{0}}$ by [29, Theorem 6.3], int $K_{t}=$ 
int $K_{t}^{0}$. Consequently, $K_{t}+n^{-1} e_{1} \subseteq K_{t}^{0}$, and finally $\zeta_{t}^{n} \in L^{0}\left(-K_{t}^{0}, \mathcal{F}_{t}\right)$ for all $t \leq T$.

Since $\zeta_{t}^{n} \in L^{0}\left(-K_{t}^{0}, \mathcal{F}_{t}\right)$ for all $t \leq T$ and $\zeta^{n} \rightarrow \xi$ as $n \rightarrow \infty$, we may assume without loss of generality that $\xi_{t} \in L^{0}\left(-K_{t}^{0}, \mathcal{F}_{t}\right)$ for all $t \leq T$. Let us define, for every $t \leq T$,

$$
\lambda^{t}:=\inf \left\{\lambda \geq 1:-\lambda \xi_{t} \in G_{t}\right\}=\inf \left\{\lambda \in \mathbb{Q} \cap[1, \infty):-\lambda \xi_{t} \in G_{t}\right\} .
$$

The second equality holds since $\mu G_{t} \subseteq G_{t}$ for all $\mu \geq 1$. We deduce that $\lambda^{t} \in L^{0}\left([1, \infty), \mathcal{F}_{t}\right)$ and $\lambda^{t} \xi^{t} \in-G_{t}$ a.s. for all $t \leq T$. Let us define

$$
\xi^{n}:=\mathbf{L}_{T}\left(\sum_{t=0}^{T} n \xi_{t} 1_{\lambda^{t} \leq n}\right), \quad n \geq 1 .
$$

Observe that on the set $\left\{\lambda^{t} \leq n\right\}$, we have $n \xi_{t} \in-G_{t}$ since $\mu G_{t} \subseteq G_{t}$ for all $\mu \geq 1$ and $t \leq T$. We deduce that $\xi^{n} \in \mathrm{A}_{T}^{0}(G)$. Moreover, a.s. for every $n$ large enough, we have $\sup _{t \leq T} \lambda^{t} \leq n$ so that $\xi^{n}=n \xi$ hence $\xi=n^{-1} \xi^{n}$. Recall that the distance in the metric space $L^{0}$ between $\xi$ and $n^{-1} \xi^{n}$ is given by

$$
d\left(\xi, n^{-1} \xi^{n}\right):=\mathbb{E}\left(\left|\xi-n^{-1} \xi^{n}\right| 1_{\sup _{t \leq T} \lambda^{t}>n} \wedge 1\right) .
$$

Therefore, by the dominated convergence theorem, $d\left(\xi, n^{-1} \xi^{n}\right) \rightarrow 0$ as $n$ goes to $\infty$ since $1_{\sup _{t \leq T} \lambda^{t}>n}=0$ if $n$ is large enough. Since $n^{-1} \xi^{n} \in{\overline{\mathrm{A}_{T}^{0}(G)}}^{0}$, we finaly deduce that $\xi \in{\overline{\mathrm{A}_{T}^{0}(G)}}^{0}$.

A natural problem arising from Theorem 3.4 is the characterization of the existence of an ESPM in the Kabanov model $K$ or, equivalently, when $G$ is a closed convex cone. In this case, the liquidation value function $x \mapsto \mathbf{L}_{t}(x)$, $t=0, \cdots, T$, is a.s. concave and $\mathrm{A}_{T}^{0}=\mathrm{LV}_{T}^{0}$. We show in the following theorem that, when $G$ is a.s. a closed convex cone, $\mathcal{D}^{1}(\mathrm{P}) \neq \emptyset$ is equivalent to the No Free Lunch condition (NFL) $\overline{\mathrm{R}}_{T}^{w} \cap L^{\infty}\left(\mathbf{R}_{+}^{d}, \mathcal{F}_{T}\right)=\{0\}$ in the approach where the attainable claims are expressed in physical units. Recall that NFL is equivalent to the existence of a Consistent Price System (CPS) $Z$, i.e. $Z$ belongs to the set $\mathcal{M}_{T}\left(K^{*} \backslash\{0\}\right)$ of all martingales with $Z_{t}$ in the positive dual cone $K_{t}^{*} \backslash\{0\}, t \leq T,[18]$.

Theorem 3.5. Assume that $G$ is a closed convex cone and $G_{T}$ strictly dominates $\mathbf{R}_{+}^{d}{ }^{4}$. The financial market model defined by $G$ satisfies the no arbitrage condition $\overline{\mathrm{LV}_{T}^{\infty}}{ }^{w} \cap L^{\infty}\left(\mathbf{R}_{+}, \mathcal{F}_{T}\right)=\{0\}$ if and only if ${\overline{\mathrm{R}_{T}^{\infty}}}^{w} \cap L^{\infty}\left(\mathbf{R}_{+}^{d}, \mathcal{F}_{T}\right)=\{0\}$.

\footnotetext{
${ }^{4}$ The set $G_{T}$ strictly dominates $\mathbf{R}_{+}^{d}$ if $\mathbf{R}_{+}^{d} \backslash\{0\} \subseteq \operatorname{int} G_{T}$.
} 
Proof. By definition of the liquidation value process $\mathbf{L}, \mathrm{A}_{T}^{\infty} e_{1} \subseteq \mathrm{R}_{T}^{\infty}$ which implies that ${\overline{\mathrm{A}_{T}^{\infty}}}^{w} e_{1} \subseteq{\overline{\mathrm{R}_{T}^{\infty}}}^{w}$. The reverse implication of the equivalence immediately follows. Suppose that $\overline{\mathrm{LV}}_{T}^{\infty} \cap L^{\infty}\left(\mathbf{R}_{+}, \mathcal{F}_{T}\right)=\{0\}$ holds. Set $\gamma:=\sup _{|x| \leq 1}\left|\mathbf{L}_{T}(x)\right|<\infty$ and let us define $d \mathrm{P}^{\prime}=\mu e^{-\gamma} d \mathrm{P}$ where $\mu>0$ is a constant such that $\mathrm{P}^{\prime}$ is a probability measure equivalent to $\mathrm{P}$. By Theorem 3.2 and Theorem 3.1, we deduce the existence of $Q \sim \mathrm{P}^{\prime}$ with $d Q / d \mathrm{P} \in L^{\infty}$ such that $\mathbb{E}_{Q} \mathbf{L}_{T}(X) \leq 0$ for every $X \in \mathrm{R}_{T}^{0}$ satisfying $\mathbf{L}_{T}(X) \in L_{Q}^{1}$. Observe that $\gamma \in L_{Q}^{p}\left(\mathbf{R}, \mathcal{F}_{T}\right)$ for every $p \geq 1$. Moreover, recall that $\mathbf{L}_{T}$ is positively homogeneous since $G$ is a cone. It follows that

$$
\left|\mathbf{L}_{T}(x)\right| \leq \gamma\|x\|, \quad \forall x \in \mathbf{R}^{d}
$$

We then have $\mathrm{R}_{T}^{\infty} \subseteq \Gamma$ where

$$
\Gamma:=\left\{X \in L^{\infty}: \mathbf{L}_{T}(X) \in L_{Q}^{1} \text { and } \mathbb{E}_{Q}\left[\mathbf{L}_{T}(X)\right] \leq 0\right\}
$$

Consider the family $\mathcal{C}$ of all convex subsets of $\Gamma$ we endow with the partial order: $C_{1} \preceq C_{2}$ if and only if $C_{1} \subseteq C_{2}$. Observe that any chain $\left(C_{i}\right)_{i \in I}$ of $\mathcal{C}$ admits the upper bound $C_{\infty}:=\cup_{i \in I} C_{i}$ which is convex since any two elements of $\left(C_{i}\right)_{i \in I}$ are comparable. Therefore, by the Zorn lemma, $\mathcal{C}$ admits a maximal element and, by the Hausdorff maximal principle, $\mathrm{R}_{T}^{\infty} \subseteq C^{*} \subseteq \Gamma$ where $C^{*}$ is a maximal convex element. Let us show that $C^{*}$ is $\sigma\left(L^{\infty}, L^{1}\right)$-closed. To do so, first consider the norm closure $\overline{C^{*}} L^{2}$ of $C^{*}$ in $L_{Q}^{2}$. Let $X \in \overline{C^{*}} L^{2}$ and let $\left(X^{n}\right)$ be a sequence of $C^{*}$ such that $X^{n}$ converges to $X$ in $L_{Q}^{2}$. We may also assume that $X^{n} \rightarrow X$ a.s. hence $\mathbf{L}_{T}\left(X^{n}\right) \rightarrow \mathbf{L}_{T}(X)$ a.s. By $(3.10), \mathbf{L}_{T}(Y) \in L_{Q}^{1}$ for every $Y \in L_{Q}^{2}\left(\mathbf{R}^{d}, \mathcal{F}_{T}\right)$. Moreover, for every $M>0$,

$$
\begin{aligned}
\mathbb{E}_{Q}\left|\mathbf{L}_{T}\left(X^{n}\right)-\mathbf{L}_{T}(X)\right| & \leq \mathbb{E}_{Q}\left|\mathbf{L}_{T}\left(X^{n}\right)-\mathbf{L}_{T}(X)\right| 1_{\left|\mathbf{L}_{T}\left(X^{n}\right)\right| \leq M} \\
& +\mathbb{E}_{Q}\left|\mathbf{L}_{T}\left(X^{n}\right)\right| 1_{\left|\mathbf{L}_{T}\left(X^{n}\right)\right|>M}+\mathbb{E}_{Q}\left|\mathbf{L}_{T}(X)\right| 1_{\left|\mathbf{L}_{T}\left(X^{n}\right)\right|>M}
\end{aligned}
$$

Applying the Cauchy-Schwarz inequality, using (3.10) and, finally, using the Bienaymé-Tchebychev inequality, we obtain that

$$
\begin{aligned}
\mathbb{E}_{Q}\left|\mathbf{L}_{T}\left(X^{n}\right)\right| 1_{\left|\mathbf{L}_{T}\left(X^{n}\right)\right|>M} & \leq \sup _{n}\left\|X^{n}\right\|_{2} \sqrt{\mathbb{E}_{Q} \gamma^{2} 1_{\left|\mathbf{L}_{T}\left(X^{n}\right)\right|>M}} \\
& \leq \sup _{n}\left\|X^{n}\right\|_{2}\|\gamma\|_{4}\left(\mathrm{P}\left(\left|\mathbf{L}_{T}\left(X^{n}\right)\right|>M\right)\right)^{1 / 4} \\
& \leq \frac{1}{M^{1 / 4}}\left(\sup _{n}\left\|X^{n}\right\|_{2}\|\gamma\|_{4}\right)^{5 / 4}
\end{aligned}
$$


Similarly, we obtain that

$$
\mathbb{E}_{Q}\left|\mathbf{L}_{T}(X)\right| 1_{\left|\mathbf{L}_{T}\left(X^{n}\right)\right|>M} \leq \frac{\|X\|_{2}}{M^{1 / 4}}\left(\sup _{n}\left\|X^{n}\right\|_{2}\right)^{1 / 4}\left(\|\gamma\|_{4}\right)^{5 / 4}
$$

Therefore, for every $\epsilon>0$, there exists $M$ large enough such that

$$
\sup _{n}\left(\mathbb{E}_{Q}\left|\mathbf{L}_{T}\left(X^{n}\right)\right| 1_{\left|\mathbf{L}_{T}\left(X^{n}\right)\right|>M}+\mathbb{E}_{Q}\left|\mathbf{L}_{T}(X)\right| 1_{\left|\mathbf{L}_{T}\left(X^{n}\right)\right|>M}\right) \leq \epsilon .
$$

Moreover, applying the dominated convergence theorem, we deduce that $\mathbb{E}_{Q}\left|\mathbf{L}_{T}\left(X^{n}\right)-\mathbf{L}_{T}(X)\right| 1_{\left|\mathbf{L}_{T}\left(X^{n}\right)\right| \leq M} \rightarrow 0$ as $n \rightarrow \infty$. Using (3.11), we finally deduce that $\mathbf{L}_{T}\left(X^{n}\right) \rightarrow \mathbf{L}_{T}(X)$ in $L_{Q}^{1}$ hence $\mathbb{E}_{Q} \mathbf{L}_{T}(X) \leq 0$. This implies that $C^{*} \subseteq{\overline{C^{*}}}^{L^{2}} \cap L^{\infty} \subseteq \Gamma$. Since $\bar{C}^{L^{2}}$ is convex and $C^{*}$ is maximal, we deduce that $C^{*}=\bar{C}^{*} L^{2} \cap L^{\infty}$.

Let us show that $C^{*}$ is $\sigma\left(L^{\infty}, L^{1}\right)$-closed. By [23, Proposition 5.5.1], it is enough to show that $C_{R}^{*}:=C^{*} \cap\left\{X \in L^{\infty}:\|X\|_{\infty} \leq R\right\}$ is closed in probability for all $R>0$. If $X^{n} \in C_{R}^{*}$ converges a.s. to $X$, then $\|X\|_{\infty} \leq$ $R$. Moreover, by the dominated convergence theorem, we also deduce that $X^{n} \rightarrow X$ in $L^{2}$. We deduce that $X \in \overline{C^{*}} L^{2} \cap L^{\infty}=C^{*}$. Therefore, $X \in C_{R}^{*}$ and the conclusion follows. Therefore, $\overline{\mathrm{R}}_{T}^{\infty} \subseteq C^{*} \subseteq \Gamma$. By definition of $\Gamma$, we then deduce that ${\overline{\mathrm{R}_{T}^{\infty}}}^{w} \cap L^{\infty}\left(\mathbf{R}_{+}^{d}, \mathcal{F}_{T}\right)=\{0\}$.

\section{NA condition for non convex financial market models}

In this section, we study the relationship between the classical absence of arbitrage opportunity NA in the market defined by $G$ and the enlarged one defined by $K$ through a new notion of absence of arbitrage opportunity called No Weak Arbitrage opportunity (NWA). This condition is motivated by the financial markets with both proportional and fixed costs, see Example 2.7. If there is an arbitrage opportunity in the model with fixed transaction costs, there is a first moment $u=t$ when the agent holding the portfolio pays the fixed $\operatorname{cost} c_{t}$ with a positive probability. Indeed, otherwise, the terminal liquidation value of the portfolio process is non positive. Therefore, the terminal value of the portfolio process generated by the same strategy but for the model without fixed transaction costs is larger than $c_{t}$ with a positive probability, i.e. it realizes what we call a weak arbitrage opportunity in the market without fixed costs. On the other hand, the fixed costs are typically bounded and do not depend on the size of the transactions. This leads to 
the fact that, if there is a weak arbitrage opportunity in the market without fixed costs, we may scale the associated strategy by a large coefficient, so that the fixed costs are negligible compared with the proportional transaction costs induced by the bid-ask spread, i.e we asymptotically obtain an arbitrage opportunity in the market with fixed costs.

Definition 4.1. In a financial market model defined by a liquidation value process $\mathbf{L}$, we say that a portfolio process $V$ starting from the initial endowment $V_{0-}=0$ realizes a weak arbitrage opportunity if there exists $t \in$ $\{0,1, \cdots, T\}$ such that $V_{u}=0$ for all $u \leq t-1$, there exists $B_{t} \in \mathcal{F}_{t}$ with $\mathrm{P}\left(B_{t}\right)>0$ such that $V_{u} 1_{\Omega \backslash B_{t}}=0$ for all $u=t, \cdots, T$ and $\mathbf{L}_{T}\left(V_{T}\right) \geq m_{t}$ on $B_{t}$ where $m_{t} \in L^{0}\left((0, \infty), \mathcal{F}_{t}\right)$. When there is no weak arbitrage opportunity, we say that the property No Weak Arbitrage (NWA) holds.

Notice that, if $\mathbf{L}_{T}\left(V_{T}\right)>m_{t}$ on $B_{t}$, then we may change the portfolio position $\mathbf{L}_{T}\left(V_{T}\right) 1_{B_{t}} e_{1}$ into the $\mathcal{F}_{t}$-measurable terminal position $m_{t} 1_{B_{t}}$. Moreover, $t$ is necessarily less than $T-1$. Indeed, otherwise $V_{T-1}=0$ hence $V_{T} \in\left(-G_{T}\right) \cap G_{T}$ and therefore $\mathbf{L}_{T}\left(V_{T}\right)=0$. The result below is immediate:

Lemma 4.2. The following conditions are equivalent:

i) Condition NWA holds,

ii) $\mathrm{LV}_{T}^{t} \cap L^{0}\left(\mathbf{R}_{+}, \mathcal{F}_{t}\right)=\{0\}$, for all $t \leq T-1$,

iii) $\mathrm{R}_{T}^{t} \cap L^{0}\left(\mathbf{R}_{+} e_{1}, \mathcal{F}_{t}\right)=\{0\}$, for all $t \leq T-1$,

iv) For all $t \leq T-1$, and every $g_{u} \in L^{0}\left(G_{u}, \mathcal{F}_{u}\right), u=t, \cdots, T$, the condition $0=g_{t}+\cdots+g_{T}$ a.s. implies that $\mathbf{L}_{t}\left(g_{t}\right)=0$.

Remark 4.3. It is immediate that Condition NWA is weaker than Condition NA. Moreover, Condition NWA is strictly weaker than Condition NA in general. Indeed, consider a finite probability space $\Omega=\left\{\omega_{1}, \omega_{2}\right\}$ such that $\mathrm{P}\left(\omega_{i}\right)=\frac{1}{2}, i=1,2$. Consider a two step frictionless financial market model, $t=0,1$, given by a bond $B_{0}=B_{1}=1$ and a risky asset $S_{0}=1, S_{1}=1+\xi$ where $\xi\left(\omega_{1}\right)=0, \xi\left(\omega_{2}\right)=1$. This model admits infinitely many arbitrage opportunities. Indeed, consider the buy and hold strategy $(-x, x), x>0$. At time $T=1$, the liquidation value is $\mathbf{L}_{T}((-x, x))=x 1_{\left\{\omega_{2}\right\}}$. However, this model satisfies NWA. Indeed, $\mathcal{F}_{0}=\{\emptyset, \Omega\}$ hence there exists a weak arbitrage opportunity if and only if there exists a constant $m>0$ and a terminal portfolio position $V_{1}=(-x, x) \in R_{1}^{0}, x \in \mathbf{R}$, such that $\mathbf{L}_{T}\left(V_{1}\right)=x 1_{\left\{\omega_{2}\right\}}>m$ a.s. which is impossible. Observe that there exists an equivalent separating probability measure given by the Dirac measure $\delta_{1} \ll \mathrm{P}$. 
Recall from (2.7) that, for all $\lambda>0$ and $z \in \mathbf{R}^{d}$,

$$
\mathbf{L}_{t}^{G}(\lambda z)=\lambda \mathbf{L}_{t}^{K}(z)-\lambda \delta_{t}(\lambda, z)
$$

where $\delta_{t}: \mathbf{R}_{+} \times \mathbf{R}^{d} \rightarrow \mathbf{R}_{+}$is non-increasing in the first argument and $\lim _{\lambda \rightarrow \infty} \delta_{t}(\lambda, z)=0$. Moreover, $\delta_{t}(\lambda, \beta z)=\delta_{t}(\lambda \beta, z)$ for all $\beta \in \mathbf{R}_{+}$. In the sequel, we consider the following conditions.

Condition $\mathbf{L}^{0}$ : The non-linear costs functions $\delta_{t}(\lambda, z), t=0, \cdots, T$ uniformly converge to zero as $\lambda$ tends to infinity in the sense that

$$
\lim _{\lambda \rightarrow \infty}\left\|\sup _{\lambda_{0} \in L^{0}\left([\lambda, \infty), \mathcal{F}_{t}\right)|z| \leq 1} \sup _{|z|}\left|\delta_{t}\left(\lambda_{0}, z\right)\right|\right\|_{\infty}=0 .
$$

The above condition is satisfied for financial market models with both fixed and proportional costs as in Example 2.7, where fixed costs are supposed to be bounded. In this case, $\delta_{t}(\lambda, z)=\frac{c_{t}}{\lambda} \leq O\left(\lambda^{-1}\right), \lambda \rightarrow \infty$. In Example 2.8, we have $\delta_{t}(\lambda, z)=\lambda^{\alpha-1}|z|^{\alpha} \beta_{t} S_{t}$ when $\lambda$ is large enough. Condition $\mathrm{L}^{0}$ is then satisfied provided that $\alpha<1$ and that the process $\beta S$ is bounded (e.g. when $\beta_{t}=\frac{\beta}{S_{t}}$, i.e. the non-linear costs depend on transaction volume). Finally, in Example 2.9, Condition $\mathrm{L}^{0}$ is always satisfied because $\delta(\lambda, z)=0$ as $\lambda$ is large enough.

The following theorem is the first main result of this section.

Theorem 4.4. Suppose that the market $G$ satisfies Condition $L^{0}$. Then, $G$ satisfies (NWA) if and only if $K$ does.

Proof. Since every $G$-portfolio is also a $K$-portfolio, we deduce that $G$ satisfies NWA if $K$ does. Conversely, suppose that $K$ does not satisfy NWA. By definition, there exists $B_{t} \in \mathcal{F}_{t}$ with $\mathrm{P}\left(B_{t}\right)>0$ and a portfolio process $\tilde{V}$ starting from $\tilde{V}_{t-}=0$ at time $t$, i.e. $\widetilde{V}_{u}=\sum_{r=t}^{u} \widetilde{\xi}_{r}$ for all $u \geq t, \widetilde{\xi}_{r} \in$ $L^{0}\left(-K_{r}, \mathcal{F}_{r}\right)$, such that $\widetilde{V}_{u} 1_{\Omega \backslash B_{t}}=0$ for every instant $u=t, \cdots, T$ and $\mathbf{L}_{T}^{K}\left(\tilde{V}_{T}\right)>m_{t}$ on $B_{t}$ where $m_{t} \in L^{0}\left((0, \infty), \mathcal{F}_{t}\right)$. Without loss of generality, we may suppose that $m_{t} \geq \varepsilon>0$ and we may replace $\tilde{V}_{T}$ by $\mathbf{L}_{T}^{K}\left(\tilde{V}_{T}\right) e_{1}$. Let us define, for $k>1$,

$$
\begin{aligned}
V_{u}^{k} & :=\sum_{r=t}^{u} \xi_{r}^{k} 1_{B_{t}}, \quad u \geq t \\
\xi_{r}^{k} & :=k \widetilde{\xi}_{r}+\mathbf{L}_{r}^{G}\left(-k \widetilde{\xi}_{r}\right) e_{1}, \quad t \leq r \leq T .
\end{aligned}
$$


Since $\mathbf{L}_{r}^{G}\left(-\xi_{r}^{k}\right)=0$ for all $r \geq t$, this implies that $V^{k}$ is a $G$-portfolio. Moreover, under Condition $\mathrm{G}^{0}$, we get that

$$
\mathbf{L}_{r}^{G}\left(-k \widetilde{\xi}_{r}\right) \geq \mathbf{L}_{r}^{K}\left(-k \widetilde{\xi}_{r}\right)-k \delta_{r}\left(k,-\widetilde{\xi}_{r}\right) \geq-k \delta_{r}\left(k,-\widetilde{\xi}_{r}\right), \quad r \geq t
$$

Therefore, $\mathbf{L}_{T}^{G}\left(V_{T}^{k}\right) \geq k\left[\mathbf{L}_{T}^{K}\left(\tilde{V}_{T}\right)-\sum_{r \geq t} \delta_{r}\left(k,-\widetilde{\xi}_{r}\right)\right]$. Moreover, by using the property $\delta_{r}(\lambda, \beta z)=\delta_{r}(\lambda \beta, z)$, we may suppose without loss of generality by the normalization $\beta(\omega)=1+\left\|\widetilde{\xi}_{r}(\omega)\right\|$ with $\beta k \geq k$ that $\left\|\widetilde{\xi}_{r}\right\|_{\infty} \leq 1$ for all $r \geq t$. Hence, by choosing $k$ large enough, since $\beta k \geq k$ and Condition $\mathrm{L}^{0}$ holds, $\left\|\sum_{r \geq t} \delta_{r}\left(k,-\widetilde{\xi}_{r}\right)\right\|_{\infty}<\frac{\epsilon}{2}$. We deduce that on the set $B_{t}$,

$$
\mathbf{L}_{T}^{G}\left(V_{T}^{k}\right) \geq k \mathbf{L}_{T}^{K}\left(\widetilde{V}_{T}\right)-k \frac{\epsilon}{2} \geq k \frac{\epsilon}{2}>0 .
$$

This implies that $V_{T}^{k}$ realizes a weak arbitrage opportunity in the market defined by $G$, i.e. $G$ does not satisfy NWA hence a contradiction.

We introduce two conditions of absence of asymptotic arbitrage opportunity which are the asymptotic versions of the conditions NA and NWA respectively.

Definition 4.5. We say that the condition No Arbitrage with Vanishing Risk (NAVR) holds if, for all sequence $\xi^{n} \in \mathrm{R}_{T}^{0}$ such that $\mathbf{L}_{T}\left(\xi^{n}\right) \geq-\beta_{n}, \beta_{n} \in \mathbf{R}$, for all $n$ where $\beta_{n} \rightarrow 0$, we have $\liminf _{n} \mathbf{L}_{T}\left(\xi^{n}\right)=0$.

The market satisfies the No weak Arbitrage with Vanishing Risk (NWAVR) condition if, for all $t=1, \ldots, T$ and all sequence $\xi^{n} \in \mathrm{R}_{t, T}^{0}$ such that $\mathbf{L}_{T}\left(\xi^{n}\right) \geq-\beta_{n}$ for all $n$ where $\beta_{n} \rightarrow 0$, the property $\liminf { }_{n} \mathbf{L}_{T}\left(\xi^{n}\right) \geq \psi_{t}$, $\psi_{t} \in L_{+}^{0}\left(\mathcal{F}_{t}\right)$, implies that $\psi_{t}=0$.

Condition $\mathbf{L}^{1}$ : There exists an adapted process $\left(c_{t}\right)_{t \leq T}$ such that $c_{t}>0$ a.s. and

$$
\mathbf{L}_{t}^{G}(x) \leq \mathbf{L}_{t}^{K}(x)-c_{t}, \forall x \notin \mathbf{R}_{+} e_{1} .
$$

Condition $\mathrm{L}^{1}$ means that the non linear costs the agent pays when liquidating a risky position is non degenerate. This condition holds for the models in Example 2.7 and Example 2.8.

Theorem 4.6. Suppose that the market $G$ satisfies Condition $L^{0}$ and Condition $L^{1}$. Then, the conditions $N A, N A V R, N W A$ and $N W A V R$ are equivalent. 
Proof. First, observe that the implications $N A V R \Rightarrow N A \Rightarrow N W A$ and $N A V R \Rightarrow N W A V R \Rightarrow N W A$ are trivial. Therefore, it suffices to show the implication $N W A \Rightarrow N A V R$. Suppose, on the contrary, that $N A V R$ does not hold. Then, there is a sequence $V_{T}^{n} \in \mathrm{R}_{T}^{0}$ such that $\mathbf{L}_{T}\left(V_{T}^{n}\right) \geq-\beta_{n}$ for all $n$ where $\beta_{n} \rightarrow 0$ and

$$
\liminf _{n} \mathbf{L}_{T}\left(V_{T}^{n}\right)=X_{T} \in L^{0}\left(\mathbf{R}_{+}, \mathcal{F}_{T}\right) \backslash\{0\} .
$$

Replacing $\beta_{n}$ by $\sup \left\{\beta_{k}: k \geq n\right\}$, we may suppose without generality that the sequence $\left(\beta_{n}\right)$ is non increasing.

Suppose that $V_{T}^{n}=\sum_{u=0}^{T} \xi_{u}^{n}$ where $\xi_{u}^{n} \in L^{0}\left(-G_{u}, \mathcal{F}_{u}\right), u \leq T$. We replace $V^{n}$ by the $G$-portfolio $\hat{V}_{t}^{n}:=\sum_{u=0}^{t} \hat{\xi}_{u}^{n}$ where $\hat{\xi}_{u}^{n}:=\xi_{u}^{n} 1_{\xi_{u}^{n} \notin-\mathbf{R}_{+} e_{1}}, u \leq T$. We have $\hat{V}_{t}^{n} \geq_{G_{t}} V_{t}^{n}, \forall t \leq T$, so that we may suppose without loss of generality that $\xi_{t}^{n}=\xi_{t}^{n} 1_{\xi_{t}^{n} \notin-\mathbf{R}_{+} e_{1}}$. By virtue of (4.13), we may choose $n_{0}$ large enough such that $\mathbf{L}_{T}\left(V_{T}^{n}\right) \not \equiv 0$ for every $n \geq n_{0}$. Let us define, with the convention $\min \emptyset=T+1$, the stopping time:

$$
\pi^{*}(n):=\min \left\{t \geq 0: \xi_{t}^{n} \neq 0\right\}
$$

and

$$
t^{*}(n):=\min \left\{t \geq 0: \mathrm{P}\left(\pi^{*}(n)=t\right)>0\right\} .
$$

Observe that, by (4.13), $t^{*}(n) \leq T$ and $V_{T}^{n}=\sum_{t=t^{*}(n)}^{T} \xi_{t}^{n}$. We may assume, by a compactness argument, that $t^{*}(n) \rightarrow t^{*} \in\{0, \cdots, T\}$. Since the sequence $\left(t^{*}(n)\right)_{n \in \mathbf{N}}$ only takes discrete values, we deduce that $t^{*}(n)=t^{*}$ for $n \geq n_{0}$ where $n_{0}$ is large enough. Set $B_{t^{*}(n)}^{n}:=\left\{\xi_{t^{*}(n)}^{n} \neq 0\right\}$ so that $\mathrm{P}\left(B_{t^{*}(n)}^{n}\right)>0$.

First case. Suppose that there exists $n^{1}$ large enough such that $c_{t^{*}}-\beta_{n^{1}}>\varepsilon$ for some $\varepsilon>0$ on a non null set of $B_{t^{*}}^{n_{1}}$ that we denote by $B_{t^{*}}$. We rewrite $V_{T}^{*}:=V_{T}^{n_{1}}=\sum_{t=0}^{T} \xi_{t}^{n_{1}}$ where $\xi_{t}^{*}:=\xi_{t}^{n_{1}} \in L^{0}\left(-G_{t}, \mathcal{F}_{t}\right), t \leq T$. We may replace without loss of generality $V_{T}^{*}$ by $\mathbf{L}_{T}\left(V_{T}^{*}\right) e_{1}$. By construction and assumption, we have $-\xi_{t^{*}}^{*} \notin \mathbf{R}_{+} e_{1}$ on $B_{t^{*}}$. Let us define

$$
\tilde{V}_{r}=\sum_{t=t^{*}}^{T}\left[\xi_{t}^{*}+\mathbf{L}_{t}^{K}\left(-\xi_{t}^{*}\right) e_{1}\right] 1_{B_{t^{*}}}, \quad t^{*} \leq r \leq T .
$$

Observe that $\tilde{V}$ is a $K$-portfolio process since $\tilde{\xi}_{t}:=\xi_{t}^{*}+\mathbf{L}_{t}^{K}\left(-\xi_{t}^{*}\right) e_{1}$ satisfies $\mathbf{L}_{t}^{K}\left(-\tilde{\xi}_{t}\right)=0$ for all $t^{*} \leq t \leq T$. Since $\xi_{t}^{*} \neq 0$ on $B_{t^{*}}$, we deduce by Condition $\mathrm{L}^{1}$ that $\mathbf{L}_{t}^{K}\left(-\xi_{t}^{*}\right) \geq \mathbf{L}_{t}^{G}\left(-\xi_{t}^{*}\right)+c_{t^{*}} \geq c_{t^{*}}$ on the event $B_{t^{*}}$. Since we suppose that $V_{T}^{*}=\mathbf{L}_{T}^{G}\left(V_{T}^{*}\right) e_{1}$ and $\mathbf{L}_{T}^{G}\left(V_{T}^{n}\right) \geq-\beta_{n}$ for all $n$, we get that

$$
\mathbf{L}_{T}^{K}\left(\tilde{V}_{T}\right) \geq\left(c_{t^{*}}-\beta_{n^{1}}\right) 1_{B_{t^{*}}} \geq \varepsilon 1_{B_{t^{*}}} .
$$


Let us now define

$$
V_{T}^{k}=\sum_{t=t^{*}}^{T}\left[k \tilde{\xi}_{t}+\mathbf{L}_{t}^{G}\left(-k \tilde{\xi}_{t}\right) e_{1}\right] .
$$

By a similar argument as in Theorem 4.4, we can find $k$ large enough such that $\mathbf{L}_{T}^{K}\left(V_{T}^{k}\right) \geq \frac{k \varepsilon}{2}>0$ on $B_{t^{*}}$, i.e. $V^{k}$ realises a weak arbitrage opportunity for the market defined by $G$. This is a contradiction.

Second case. For $n \geq n_{0}$, suppose that $c_{t^{*}} 1_{B_{t^{*}}^{n}} \leq \beta_{n} 1_{B_{t^{*}}^{n}}$ a.s. Since $c_{t^{*}}>0$, we deduce that $1_{B_{t^{*}}^{n}}(\omega)=0$ a.s. $(\omega)$ for every $n \geq \tilde{n}_{0}(\omega)$ and some $\tilde{n}_{0}(\omega) \in \mathbf{N}$. Let us define the sequence $\left(\tilde{n}_{m}\right)_{m \geq 1}$ of $\mathcal{F}_{t^{*}}$-measurable integer-valued random variables:

$$
\tilde{n}_{m}(\omega):=\inf \left\{\tilde{n}_{0} \geq\left(n_{0} \vee m\right): 1_{B_{t^{*}(n)}^{n}}(\omega)=0, \forall n \geq \tilde{n}_{0}\right\}<\infty,
$$

and let us define

$$
V_{T}^{m, t^{*}+1}:=V_{T}^{\tilde{n}_{m}}=\sum_{t=t^{*}}^{T} \xi_{t}^{\tilde{n}_{m}}=\sum_{t=t^{*}+1}^{T} \xi_{t}^{\tilde{n}_{m}} .
$$

Observe that for all $t \geq t^{*}, \xi_{t}^{\tilde{n}_{m}}=\sum_{k=m}^{\infty} \xi_{t}^{k} 1_{\tilde{n}_{m}=k} \in L^{0}\left(-G_{t}, \mathcal{F}_{t}\right)$. Moreover, since $\left(\beta_{n}\right)$ is decreasing, $\mathbf{L}_{T}\left(V_{T}^{m, t^{*}+1}\right)=\sum_{k=m}^{\infty} \mathbf{L}_{T}\left(V_{T}^{k} 1_{\tilde{n}_{m}=k}\right) \geq-\beta_{m}$. Clearly, we also have $\liminf _{n} \mathbf{L}_{T}\left(V_{T}^{m, t^{*}+1}\right) \geq \liminf _{n} \mathbf{L}_{T}\left(V_{T}^{n}\right) \in L^{0}\left(\mathbf{R}_{+}, \mathcal{F}_{T}\right) \backslash\{0\}$. As $\xi_{t^{*}}^{\tilde{n}_{m}}=0$, the initial situation is reproduced with the new sequence $V_{T}^{m, t^{*}+1}$. We then repeat the whole procedure and conclude with the first case by a contradiction. Indeed, otherwise, we construct similarly step by step $V_{T}^{m, u}$, $u \geq t^{*}+2$. When $u=T$, the assumption of the first case holds. In the contrary case, we get that $1_{B_{T}^{n}} \rightarrow 0$, i.e. $\xi_{T}^{n} \rightarrow 0$. This implies that the terminal liquidation value $\mathbf{L}_{T}\left(V_{T}^{n}\right)=V_{T}^{n} \cdot e_{1}=\xi_{T}^{n} \cdot e_{1} \rightarrow 0$ in contradiction with the property that $\liminf _{n} \mathbf{L}_{T}\left(V_{T}^{n}\right) \geq \mathbf{L}_{T}\left(X_{T}\right) \geq 0, \mathbf{L}_{T}\left(X_{T}\right) \neq 0$. This shows that NWA $\Rightarrow$ NAVR and the lemma is proven.

By Theorem 4.4, we deduce the following result which says that the classical no-arbitrage condition NA for a non convex model including fixed costs holds if and only if the same market with only the proportional transaction costs satisfies a weak absence of arbitrage opportunity.

Corollary 4.7. Suppose that the market $G$ satisfies Condition $L^{0}$ and Condition $L^{1}$. Then, NA holds for $G$ if and only if the condition NWA holds for the Kabanov model $K$ with only proportional transaction costs. 
Let us examine the NWA condition for the Kabanov model. In the case where $\Omega$ is a finite probability space, the condition NWA for the Kabanov model $G=K$, i.e. $\mathrm{R}_{T}^{t} \cap L^{0}\left(\mathbf{R}_{+} e_{1}, \mathcal{F}_{t}\right)=\{0\}$, for all $t \leq T-1$, trivially implies that ${\overline{\mathrm{R}_{T}^{t}}}^{0} \cap L^{0}\left(\mathbf{R}_{+} e_{1}, \mathcal{F}_{t}\right)=\{0\}$ for all $t \leq T-1$. Therefore, adapting the proof of the Kreps-Yan theorem, [18, Theorem 3.1], there exists for all $t \leq T$ a consistent price system in the class $\widetilde{\mathcal{M}}_{t}^{T}\left(G^{*}\right)=\widetilde{\mathcal{M}}_{t}^{T}\left(G^{*}, \mathrm{P}\right)$ of all P-martingales $\left(Z_{u}\right)_{u \geq t}$ such that $Z_{u} \in L\left(G_{u}^{*}, \mathcal{F}_{u}\right)$ for all $u \geq t$ and $Z_{t} \in$ $G_{t}^{*} \backslash\{0\}$ a.s. Reciprocally, by the proof of [23, Lemma 3.2.4], for every $\widetilde{\mathrm{P}} \sim \mathrm{P}$, there exists a bounded element $Z^{\infty}$ in $\widetilde{\mathcal{M}}_{t}^{T}\left(G^{*}, \widetilde{\mathrm{P}}\right), t \leq T$. We deduce that NWA holds. Indeed, if $\xi \in \mathrm{R}_{T}^{t}(K) \cap L^{0}\left(\mathbf{R}_{+} e_{1}, \mathcal{F}_{t}\right)$, we may suppose that $\xi$ is integrable under some $\widetilde{\mathrm{P}} \sim \mathrm{P}$. As $\mathbb{E}_{\widetilde{\mathrm{P}}} Z_{T}^{\infty} \xi \leq 0$ by duality, we get that $\mathbb{E}_{\widetilde{\mathrm{P}}} Z_{T}^{\infty} \xi=\mathbb{E}_{\widetilde{\mathrm{P}}} Z_{t}^{\infty} \xi=0$. Since $Z_{t}^{\infty} \in G_{t}^{*} \backslash\{0\} \subseteq \mathbf{R}_{++}^{d}$, we finally deduce that $\xi=0$.

More generally, we show below that this result still holds in the two dimensional case even if $\Omega$ is infinite by following the proof of the Grigoriev theorem [23, Theorem 3.2.15]. Recall that $\mathcal{M}_{t}^{T}\left(G^{*} \backslash\{0\}, \mathrm{P}\right), t \leq T$, is the class of consistent price systems $Z$, i.e. P-martingales such that $Z_{u} \in G_{u}^{*} \backslash\{0\}$ a.s. for all $u \in[t, T]$.

Theorem 4.8. Let $d=2$. Consider the Kabanov financial market model defined by a proper cone $G$ such that $\mathbf{R}_{+}^{d} \backslash\{0\} \subseteq$ int $G$. Then, the following statements are equivalent:

i) Condition NWA holds,

ii) ${\overline{\mathrm{R}_{T}^{t}}}^{0} \cap L^{0}\left(\mathbf{R}_{+} e_{1}, \mathcal{F}_{t}\right)=\{0\}$, for all $t \leq T-1$,

iii) For every $t \leq T-1, \widetilde{\mathcal{M}}_{t}^{T}\left(G^{*}, \mathrm{P}\right) \neq \emptyset$,

iv) For every $t \leq T-1$ and $\widetilde{\mathrm{P}} \sim \mathrm{P}$, there exists a bounded $Z \in \widetilde{\mathcal{M}}_{t}^{T}\left(G^{*}, \widetilde{\mathrm{P}}\right)$,

v) $\mathcal{M}_{t}^{T}\left(G^{*} \backslash\{0\}, \mathrm{P}\right) \neq \emptyset$ i.e. NFL holds,

vi) There is an equivalent separating probability measure, i.e. $\mathcal{D}^{1}(\mathrm{P}) \neq \emptyset$.

Proof. To show the equivalence between the fourth properties, we adapt the proof of [23, Theorem 3.2.15], using the same notations, but we write $\mathrm{R}_{T}^{t}$ in place of $\mathrm{A}_{T}^{t}$ and we work with NWA instead of $\mathrm{NA}^{w}$. The most delicate implication is (i) $\Rightarrow$ (ii). To do so, we replace the set denoted by $\Gamma_{1}^{T}$ in $[23$, Theorem 3.2.15] by

$$
\Gamma_{1}^{T}:=\left\{\xi \in L^{\infty}\left(\mathbf{R} e_{1}, \mathcal{F}_{0}\right): \mathbb{E}_{\mathrm{P}} \xi Z_{1} \leq 0, \quad \forall Z \in \widetilde{\mathcal{M}}_{t}^{T}\left(G^{*}, \mathrm{P}\right)\right\}
$$


Recall that in the definition below, $\mathcal{F}_{0}$ could be different from the trivial $\sigma$-algebra as we use an induction argument based on the number of steps. Moreover, the proposition [23, Proposition 3.2.16] is replaced by the following:

Proposition 4.9. Suppose that $\left(G_{t}\right)_{t=0, \cdots, T}$ satisfies the $N W A$ property. Then,

(a) $\Gamma_{1}^{T} \subseteq \mathrm{R}_{T}^{1}$

(b) If $\xi \in \Gamma_{1}^{T}$ is such that $\mathbb{E}_{\mathrm{P}} \xi Z_{1}<0$, for all $Z \in \widetilde{\mathcal{M}}_{t}^{T}\left(G^{*}, \mathrm{P}\right)$, then there is $\epsilon \in L^{0}\left(\mathbf{R}^{+} e_{1}, \mathcal{F}_{0}\right), \epsilon \neq 0$, such that $\xi+\epsilon \in \mathrm{R}_{T}^{1}$.

Let us introduce the condition $T_{N}, N \geq 1$, meaning that Theorem 4.8 holds for a $N+1$ steps model $\left(G_{t_{i}}\right)_{i=0, \cdots, N}$ with $t_{i} \in 0, \cdots, T$ and $G$ may be different from the initial cone of the model. The condition $P_{N}$ means that Proposition 4.9 holds for a $N+1$ steps model. It is clear that $P_{1}$ and $T_{1}$ holds. Following the arguments of [23, Theorem 3.2.15], we show the implication $\left(T_{N}, P_{N+1}\right) \Rightarrow T_{N+1}$. We also need to show that $\left(T_{N+1}, P_{N+1}\right) \Rightarrow P_{N+2}$. To do so, we follow the same idea. The condition (a) of $P_{N+2}$ is similarly shown. For the condition (b), the first and second cases in [23, Theorem 3.2.15], i.e. Case 1 and 2 , are respectively replaced by the assumption that the $\mathcal{F}_{0^{-}}$ measurable sets $\left\{\xi \in \operatorname{int} G_{0}\right\}$ and $\left\{\xi \in-\operatorname{int} G_{0}\right\}$ are non null. Following the same arguments, we conclude in the first case by increasing $\xi$ by an $\mathcal{F}_{0^{-}}$ measurable random variable as $\left\{\xi \in \operatorname{int} G_{0}\right\} \in \mathcal{F}_{0}$. The second case appears to be impossible because, in the contrary case, there exists $\theta \in L^{0}\left(\mathbf{R}_{+} e_{1}, \mathcal{F}_{0}\right)$, $\theta \neq 0$ on $\Gamma$, such that $\xi-\theta \in L^{0}\left(G_{0}, \mathcal{F}_{0}\right)$. So, as $\xi \in \mathrm{R}_{N+2}^{1}$, we deduce that $\theta \in \mathrm{R}_{N+2}^{0}$ in contradiction with NWA for the $N+3$ steps model. Therefore, we may suppose that $P\left(\left\{\xi \in \operatorname{int} G_{0}\right\}\right)=P\left(\left\{\xi \in-\operatorname{int} G_{0}\right\}\right)=0$ and we deduce that the components of $\xi$ have different signs. By Case 2 of $[23$, Theorem 3.2.15], we also obtain that $P\left(\left\{\xi \in-\operatorname{int} G_{1}\right\}\right)=0$. Then, we proceed as in Case 3 of [23, Theorem 3.2.15]. It is worth noticing that in this proof, the random set $\widetilde{G}_{1}=\mathbf{R} \xi+\mathbf{R}_{+}^{2}$ is $\mathcal{F}_{0}$-measurable. So, as the same arguments shows that NWA does not hold for the $N+2$ steps model $\widetilde{G}$, we apply by the induction hypothesis Theorem 4.8 with the filtration $\left(\mathcal{F}_{0}, \mathcal{F}_{2}, \cdots, \mathcal{F}_{N+2}\right)$, $\widetilde{G}_{1}$ being $\mathcal{F}_{0}$-measurable. We then find $\epsilon \in L^{0}\left(\mathbf{R}_{+} e_{1}, \mathcal{F}_{0}\right), \epsilon \neq 0$, such that $\epsilon \in \mathrm{R}_{N+1}^{1}(\widetilde{G})$. We then follow the proof of [23, Theorem 3.2.15]. As any $\beta \in L^{0}\left(\mathbf{R}_{+}^{2}, \mathcal{F}_{0}\right)$ may be changed into $\mathbf{L}_{0}(\beta) e_{1} \in L^{0}\left(\mathbf{R}_{+} e_{1}, \mathcal{F}_{0}\right)$, we then conclude as in [23, Theorem 3.2.15].

Notice that in the proof $\left(T_{N}, P_{N+1}\right) \Rightarrow T_{N+1}$, Case 2 is excluded. If we 
have a detailed look on Case 1 , an element $Z \in \widetilde{\mathcal{M}}_{t}^{T}\left(G^{*}, P\right)$ is constructed as $Z_{u}=\sum_{i} 2^{-i} 1_{\Delta_{i}} \mathbb{E}\left(Z_{T}^{i} \mid \mathcal{F}_{u}\right), u \in[t, T]$ where $\Delta_{i} \in \mathcal{F}_{t}$ and $Z^{i} \in \widetilde{\mathcal{M}}_{t+1}^{T}\left(G^{*}, P\right)$ for all $i \in \mathbf{N}$. In particular, $Z_{t}=\sum_{i} 2^{-i} 1_{\Delta_{i}} \mathbb{E}\left(Z_{t+1}^{i} \mid \mathcal{F}_{t}\right) \in G_{t}^{*}$ a.s. By definition, $Z_{t+1}^{i} \in G_{t+1}^{*} \backslash\{0\}$ a.s. hence $Z_{t+1}^{i} \in \mathbf{R}_{++}^{2}$ a.s. It follows that $\mathbb{E}\left(Z_{t+1}^{i} \mid \mathcal{F}_{t}\right) \in$ $\mathbf{R}_{++}^{2}$ a.s. hence $Z_{t} \in G_{t}^{*} \backslash\{0\}$ a.s. Therefore, starting from $Z_{T}$ in the set $\widetilde{\mathcal{M}}_{T}^{T}\left(G^{*}, \mathrm{P}\right)=\mathcal{M}_{T}^{T}\left(G^{*} \backslash\{0\}, \mathrm{P}\right)=L^{0}\left(G_{T}^{*} \backslash\{0\}, \mathrm{P}\right)$, we may construct backward a consistent price system $Z \in \mathcal{M}_{t}^{T}\left(G^{*} \backslash\{0\}, \mathrm{P}\right) \neq \emptyset$. We deduce that $\mathrm{NWA} \Rightarrow(\mathrm{v})$. Since (v) implies (iii), the five statements are equivalent. At last, (v) and (vi) are equivalent by virtue of Theorem 3.5.

\section{European option pricing}

In the Kabanov market model with proportional transaction costs, contingent claims are vector-valued random variables, i.e. they are expressed in physical units as in our paper. Using convexity, the superhedging prices of an European option are dually characterized by the set of consistent price systems, see for example [22]. For non convex models, an alternative approach is suggested in [25].

When dealing with a liquidation value process, it is natural to suppose that an European claim is a terminal wealth expressed in cash. Similarly, it is also natural to seek for the minimal amount expressed in cash (the bond defining the first position of the portfolio processes, e.g. a currency) allowing to start a portfolio process ending up with a terminal value the liquidation value of which is greater than the payoff. This approach makes sense as it allows to take into account liquidation costs at both initial and final trading dates.

Let $\xi \in L^{0}\left(\mathbf{R}, \mathcal{F}_{T}\right)$ be a real-valued European contingent claim which is bounded from below. Let us define

$$
\Gamma_{\xi}=\left\{x \in \mathbf{R} \mid \exists V_{T} \in R_{T}^{0}: x+\mathbf{L}_{T}\left(V_{T}\right) \geq \xi\right\} .
$$

We define the super hedging price of the contingent claim $\xi$ as $V_{0}^{\xi}:=\inf \Gamma_{\xi}$.

In the case where $G$ is the closed convex cone of the Kabanov model, consider

$$
\mathcal{Q}^{1}(\mathrm{P}):=\left\{Q \sim \mathrm{P}: \frac{d Q}{d \mathrm{P}}=Z_{T}^{1}, Z \in \mathcal{M}_{T}^{0}\left(G^{*} \backslash\{0\}, \mathrm{P}\right), Z_{0}^{1}=1\right\},
$$


where $G^{*}$ is the positive dual of $G$ and $\mathcal{M}_{T}^{0}\left(G^{*} \backslash\{0\}, \mathrm{P}\right)$ is the set of all Pmartingales $Z$ (called consistent price systems, CPS) such that $Z_{t} \in G_{t}^{*} \backslash\{0\}$ for all $t=0, \cdots, T$, see Chapter 3 [23]. When $Z \in \mathcal{M}_{T}^{0}\left(G^{*} \backslash\{0\}\right)$ satisfies $Z_{t} \in \operatorname{int} G_{t}^{*}$, we say that $Z$ is a strictly CPS. By virtue of Lemma 3.3.2. [23], observe that $\mathcal{Q}^{1}(\mathrm{P}) \subseteq \mathcal{D}^{1}(\mathrm{P})$ where $\mathcal{D}^{1}$ is given by (3.8). Therefore,

$$
V_{0}^{\xi} \geq \sup \left\{\mathbb{E}_{Q} \xi: Q \in \mathcal{D}^{1}(\mathrm{P})\right\} \geq \sup \left\{\mathbb{E}_{Q} \xi: Q \in \mathcal{Q}^{1}(\mathrm{P})\right\}
$$

When the market is convex and there exists a strictly consistent price systems, the superhedging price of an European option is given by the following result.

Theorem 5.1. Assume that $G$ is the solvency cone in the discrete-time Kabanov model. Suppose that $\mathcal{M}_{T}^{0}\left(\operatorname{int} G^{*}, \mathrm{P}\right) \neq \emptyset$. Then, $\mathcal{D}^{1}(\mathrm{P}) \neq \emptyset$ and

$$
V_{0}^{\xi}=\sup _{Q \in \mathcal{D}^{1}(\mathrm{P})} \mathbb{E}_{Q} \xi=\sup _{Q \in \mathcal{Q}^{1}(\mathrm{P})} \mathbb{E}_{Q} \xi
$$

Proof. It suffices to verify that $V_{0}^{\xi}=\sup _{Q \in \mathcal{Q}^{1}(\mathrm{P})} \mathbb{E}_{Q} \xi$. To do so, observe that

$$
\Gamma_{\xi}=\left\{x \in \mathbf{R}: \exists V_{T} \in R_{T}^{0}: x e_{1}+V_{T} \in \xi e_{1}+G_{T}\right\} .
$$

Define

$$
D_{\xi}:=\left\{x \in \mathbf{R}: Z_{0}^{1} x \geq \mathbb{E} Z_{T}^{1} \xi, \forall Z \in \mathcal{M}_{T}^{0}\left(G^{*} \backslash\{0\}\right)\right\} .
$$

Since $\mathcal{M}_{T}^{0}\left(\right.$ int $\left.G^{*}\right) \neq \emptyset$, we adapt the proof of Theorem 3.3.3 [23] and deduce that the set $R_{T}^{0}$ is closed in probability and $\Gamma_{\xi}=D_{\xi}$. Therefore, we have $V_{0}^{\xi}=\inf D_{\xi}=\sup _{Q \in \mathcal{Q}^{1}} \mathbb{E}_{Q} \xi$.

The theorem above gives a characterization of the superhedging price of an European option based on the set of separating measures in a conical model. However, the proof relies on convex analysis and therefore cannot be extended to non convex markets. Nevertheless, we may link the price in the non convex market to that of the enlarged one which is conical. Precisely, we show under an extra condition that prices in the two markets are asymptotically equal when hedging a large number of the same European claim.

Recall from (2.7) that, for all $\lambda>0$ and $z \in \mathbf{R}^{d}$,

$$
\mathbf{L}_{t}^{G}(\lambda z)=\lambda \mathbf{L}_{t}^{K}(z)-\lambda \delta_{t}(\lambda, z)
$$


where $\delta_{t}: \mathbf{R}_{+} \times \mathbf{R}^{d} \rightarrow \mathbf{R}_{+}$is non-increasing in the first argument and $\lim _{\lambda \rightarrow \infty} \delta_{t}(\lambda, z)=0$. Moreover, $\delta_{t}(\lambda, \beta z)=\delta_{t}(\lambda \beta, z)$ for all $\beta \in \mathbf{R}_{+}$.

The following theorem extends the results of [15] where only fixed costs are considered. In particular, the option price function appears to satisfy a similar property than that of the liquidation function.

Theorem 5.2. Assume that the market $G$ satisfies Condition $G^{0}$ and the liquidation process $\mathbf{L}$ satisfies Condition $L^{0}$. Let $V_{0}^{G, \xi}$ and $V_{0}^{K, \xi}$ be the superhedging prices for the European claim $\xi$ with respect to the models defined by $G$ and $K=\overline{\mathbf{R}_{+} G}$ respectively. We have the following decomposition

$$
V_{0}^{G, \lambda \xi}=\lambda V_{0}^{K, \xi}-\lambda \sigma(\lambda, \xi)
$$

where $\sigma(\lambda, \xi)$ is non-increasing in $\lambda$ and $\lim _{\lambda \rightarrow \infty} \sigma(\lambda, \xi)=0$.

Proof. We prove that

$$
V_{0}^{K, \xi}=\lim _{\lambda \rightarrow \infty} \frac{V_{0}^{G, \lambda \xi}}{\lambda},
$$

where $\lambda \mapsto V_{0}^{G, \lambda \xi} \lambda^{-1}$ is non-increasing in $\lambda$. First, notice that if $\lambda \geq 1$ and $x>V_{0}^{G, \xi}$ then, by definition of the super hedging price, there exists a portfolio process $V$ starting from the zero initial endowment such that

$$
x+\mathbf{L}_{T}\left(V_{T}\right) \geq \xi
$$

Therefore,

$$
\lambda x+\mathbf{L}_{T}\left(\lambda V_{T}\right) \geq \lambda x+\lambda \mathbf{L}_{T}\left(V_{T}\right) \geq \lambda \xi, \quad \lambda \geq 1 .
$$

Consequently, $\lambda x \geq V_{0}^{G, \lambda \xi}$ and, finally, we have

$$
V_{0}^{G, \xi} \geq \frac{V_{0}^{G, \lambda \xi}}{\lambda}, \quad \forall \lambda \geq 1 .
$$

A similar argument allows to conclude that the function $\lambda \mapsto V_{0}^{G, \lambda \xi} \lambda^{-1}$ is non-increasing. We now prove that the limit of this function at infinity is $V_{0}^{K, \xi}$. Notice that $V_{0}^{G, \lambda \xi} \geq V_{0}^{K, \lambda \xi}=\lambda V_{0}^{G, \xi}$. Hence, the limit is at least $V_{0}^{K, \xi}$. If $x>V_{0}^{K, \xi}$, there exists a $K$-portfolio $\widetilde{V}_{T}=\sum_{k=1}^{T} \widetilde{\xi}_{k}$, with $\widetilde{\xi}_{t} \in L^{0}\left(-K_{t}, \mathcal{F}_{t}\right)$, such that $x+\mathbf{L}_{T}^{K}\left(\widetilde{V}_{T}\right) \geq \xi$. Let us define, for $\lambda \geq 1$,

$$
\begin{aligned}
V_{t}^{\lambda} & :=\sum_{u=0}^{t} \xi_{u}^{\lambda}, \quad 0 \leq t \leq T \\
\xi_{t}^{\lambda} & :=\lambda \widetilde{\xi}_{t}+\mathbf{L}_{t}^{G}\left(-\lambda \widetilde{\xi}_{t}\right) e_{1}, \quad 0 \leq t \leq T .
\end{aligned}
$$


Since $\mathbf{L}_{t}^{G}\left(-\xi_{t}^{\lambda}\right)=0$, this implies that $V^{\lambda}$ is a $G$-portfolio. Moreover

$$
\begin{aligned}
\lambda(x+\varepsilon)+\mathbf{L}_{T}^{G}\left(V_{T}^{\lambda}\right) & =\lambda(x+\varepsilon)+\mathbf{L}_{T}^{G}\left(\lambda \widetilde{V}_{T}\right)+\sum_{t=0}^{T} \mathbf{L}_{t}^{G}\left(-\lambda \widetilde{\xi}_{t}\right) \\
& =\lambda(x+\varepsilon)+\lambda \mathbf{L}_{T}^{K}\left(\widetilde{V}_{T}\right)-\lambda \delta_{T}\left(\lambda, \widetilde{V}_{T}\right) \\
& +\lambda \sum_{t=0}^{T} \mathbf{L}_{t}^{K}\left(-\widetilde{\xi}_{t}\right)-\lambda \sum_{t=0}^{T} \delta_{t}\left(\lambda,-\widetilde{\xi}_{t}\right) \\
& =\lambda\left(x+\mathbf{L}_{T}^{K}\left(\widetilde{V}_{T}\right)\right)+\lambda\left(\varepsilon-\delta_{T}\left(\lambda, \widetilde{V}_{T}\right)-\sum_{t=0}^{T} \delta_{t}\left(\lambda,-\widetilde{\xi}_{t}\right)\right) \\
& +\lambda \sum_{t=0}^{T} \mathbf{L}_{t}^{K}\left(-\widetilde{\xi}_{t}\right) .
\end{aligned}
$$

Notice that $\sum_{t=0}^{T} \mathbf{L}_{t}^{K}\left(-\widetilde{\xi}_{t}\right) \geq 0$. Moreover, by using the property $\delta_{r}(\lambda, \beta z)=$ $\delta_{r}(\lambda \beta, z)$, we may suppose without loss of generality by the normalization $\beta(\omega)=1+\left\|\widetilde{V}_{T}(\omega)\right\|$ with $\beta \lambda \geq \lambda$ that $\left\|\widetilde{V}_{T}\right\|_{\infty} \leq 1$ for all $t \leq T$ (and similarly for $\xi_{t}$ ). Hence, by choosing $\lambda$ large enough and applying Condition $\mathrm{L}^{0}$, we have

$$
\left\|\sum_{t \leq T} \delta_{t}\left(\lambda,-\widetilde{\xi}_{t}\right)+\delta_{T}\left(\lambda, \widetilde{V_{T}}\right)\right\|_{\infty}<\varepsilon .
$$

Consequently,

$$
\lambda(x+\varepsilon)+\mathbf{L}_{T}^{G}\left(V_{T}^{\lambda}\right) \geq \lambda\left(x+\mathbf{L}_{T}^{K}\left(\widetilde{V}_{T}\right)\right) \geq \lambda \xi
$$

This implies that $\lambda(x+\varepsilon) \geq V_{0}^{G, \lambda \xi}$ for $\lambda$ large enough, or equivalently

$$
\lim _{\lambda \rightarrow \infty} \frac{V_{0}^{G, \lambda \xi}}{\lambda} \leq x+\varepsilon, \quad \forall x>V_{0}^{K, \xi}, \forall \varepsilon>0 .
$$

This implies that $\lim _{\lambda \rightarrow \infty} \frac{V_{0}^{G, \lambda \xi}}{\lambda} \leq V_{0}^{K, \xi}$ and the conclusion follows.

\section{Appendix}

\section{Proof of Proposition 2.3}

1. We first prove that if $A$ is an arbitrary set in $\mathbf{R}^{d}$ such that $A+\mathbf{R}_{+} e_{1} \subseteq A$ and $f: \mathbf{R}^{d} \rightarrow \mathbf{R} \cup\{\infty\}$ satisfies the following conditions: 
(i) $f(z) \geq 0, \forall z \in A$.

(ii) $f(x+y) \geq f(x)+f(y), \forall x, y \in \mathbf{R}^{d}$.

(iii) $f\left(\alpha e_{1}\right) \geq \alpha, \forall \alpha \in \mathbf{R}$.

(iv) $|f(z)|<\infty$ implies $z-f(z) e_{1} \in A, \forall z \in \mathbf{R}^{d}$,

then $f$ is uniquely defined. Indeed, suppose there exists two functions $f_{1}, f_{2}$ satisfying Conditions (i)-(iv). Since $A+\mathbf{R}_{+} e_{1} \subseteq A, z-k e_{1} \in A$ for all $k<f_{2}(z)$ and we get that

$$
f_{1}(z) \geq f_{1}\left(z-k e_{1}\right)+f_{1}\left(k e_{1}\right) \geq k
$$

We deduce that $f_{1}(z) \geq f_{2}(z)$ as $k \rightarrow f_{2}(z)$ and, by symmetry, we deduce that $f_{1}=f_{2}$.

Let us verify that $\widetilde{\mathbf{L}}_{t}$ satisfies Conditions (i)-(iv) with $\widetilde{G}_{t}$ in place of $A$. The conditions (i)-(ii) are easily seen using the definition of $\widetilde{\mathbf{L}}_{t}$ and the property $\mathbf{L}_{t}(0)=0$. Since $\widetilde{\mathbf{L}}_{t}$ is super-additive, we have $\widetilde{\mathbf{L}}_{t}(0) \leq 0$. Moreover, $\widetilde{\mathbf{L}}_{t}(0) \geq$ $\mathbf{L}_{t}(0)=0$ hence $\widetilde{\mathbf{L}}_{t}(0)=0$. By the super-additivity condition, we also have, for all $\alpha \in \mathbf{R}$,

$$
\widetilde{\mathbf{L}}_{t}(0)=0 \geq \widetilde{\mathbf{L}}_{t}\left(-\alpha e_{1}\right)+\widetilde{\mathbf{L}}_{t}\left(\alpha e_{1}\right) \geq \mathbf{L}_{t}\left(-\alpha e_{1}\right)+\mathbf{L}_{t}\left(\alpha e_{1}\right)=0 .
$$

This implies that $\widetilde{\mathbf{L}}_{t}\left(-\alpha e_{1}\right)=-\widetilde{\mathbf{L}}_{t}\left(\alpha e_{1}\right)$ for all $\alpha$. As $\widetilde{\mathbf{L}}_{t}\left(\alpha e_{1}\right) \geq \mathbf{L}_{t}\left(\alpha e_{1}\right)=\alpha$ for all $\alpha, \widetilde{\mathbf{L}}_{t}\left(\alpha e_{1}\right)=-\widetilde{\mathbf{L}}_{t}\left(-\alpha e_{1}\right) \leq \alpha$ and finally $\widetilde{\mathbf{L}}_{t}\left(\alpha e_{1}\right)=\alpha$ i.e. (iii) holds. The condition (iv) follows from the inequality

$$
\widetilde{\mathbf{L}}_{t}\left(x-\widetilde{\mathbf{L}}_{t}(x) e_{1}\right) \geq \widetilde{\mathbf{L}}_{t}(x)+\widetilde{\mathbf{L}}_{t}\left(-\widetilde{\mathbf{L}}_{t}(x) e_{1}\right)=0, x \in \mathbf{R}^{d} .
$$

Similarly, we get that $\widetilde{\mathbf{L}}_{t}\left(x+\alpha e_{1}\right) \geq \widetilde{\mathbf{L}}_{t}(x)+\alpha$ for all $\alpha \in \mathbf{R}$ hence the inclusion $\widetilde{G}_{t}+\mathbf{R}_{+} e_{1} \subseteq \widetilde{G}_{t}$ holds. Let us define

$$
\widetilde{L}_{t}(z):=\sup \left\{\alpha \in \mathbf{R}: z-\alpha e_{1} \in \widetilde{G}_{t}\right\}
$$

It suffices to show that $\widetilde{L}_{t}$ also satisfies the conditions (i)-(iv) above. The condition (i) is deduced directly from the definition of $\widetilde{L}_{t}$.

Since $\widetilde{G}_{t}$ is closed, the condition (iv) is satisfied. Moreover, by definition of $\widetilde{L}_{t}$, we have $\widetilde{L}_{t}\left(x+\alpha e_{1}\right)=\widetilde{L}_{t}(x)+\alpha$. Therefore, as $x-k^{x} e_{1}, y-k^{y} e_{1} \in \widetilde{G}_{t}$ for all $k^{x}<\widetilde{L}_{t}(x)$ and $k^{y}<\widetilde{L}_{t}(y)$, we get that

$$
\widetilde{L}_{t}(x+y)-k^{x}-k^{y}=\widetilde{L}_{t}\left(x-k^{x} e_{1}+y-k^{y} e_{1}\right) \geq 0 .
$$


The condition (ii) then follows by making $k^{x}$ and $k^{y}$ converged to $\widetilde{L}_{t}(x)$ and $\widetilde{L}_{t}(y)$ respectively. Consequently, $\widetilde{L}_{t}(0) \leq 0$ which implies that $\widetilde{L}_{t}(0)=0$ and then $\widetilde{L}_{t}\left(\alpha e_{1}\right)=\alpha$. The condition (iii) is satisfied. Since the two functions $\widetilde{L}_{t}$ and $\widetilde{\mathbf{L}}_{t}$ satisfies the conditions (i)-(iv), they coincide as shown above.

It is clear that the random set $\widetilde{G}$ satisfies Condition $G^{0}$ (i)-(iv).

2. Let us first define

$$
\widehat{\mathbf{L}}_{t}(z):=\sup \left\{\alpha \in \mathbf{R}: z-\alpha e_{1} \in \widehat{G}_{t}\right\}
$$

We have

$$
\widehat{\mathbf{L}}_{t}(z) \geq \widehat{\mathbf{L}}_{t}\left(z-\mathbf{L}_{t}(z) e_{1}\right)+\widehat{\mathbf{L}}_{t}\left(\mathbf{L}_{t}(z) e_{1}\right) \geq \mathbf{L}_{t}(z)
$$

Therefore, $\widehat{\mathbf{L}}_{t} \geq \mathbf{L}_{t}$. Moreover, since $\widehat{G_{t}}$ satisfies Conditions $G^{0}$ (i)-(iv), we deduce that $\widehat{\mathbf{L}}_{t}$ satisfies the conditions (i)-(iv) introduced in the beginning of the proof. But $\widetilde{\mathbf{L}}_{t}$ is the smallest function dominating $\mathbf{L}_{t}$ and satisfying these properties and $\widehat{\mathbf{L}}_{t} \leq \widetilde{\mathbf{L}}_{t}$ ( since $\widehat{G}_{t} \subseteq \widetilde{G}_{t}$ ). Therefore, $\widehat{\mathbf{L}}_{t}=\widetilde{\mathbf{L}}_{t}$ hence $\widetilde{G}_{t}=\widehat{G}_{t}$ (notice that $\left\{\widehat{\mathbf{L}}_{t}=0\right\} \subseteq \partial \widehat{G_{t}}$, see [25], Proposition 2.6.).

\section{Proof of Proposition 2.4}

(i) We first prove that $D_{+} \mathbf{L}_{t}(0)$ is super-additive, homogeneous of degree one and dominates $\mathbf{L}_{t}$. Indeed, since $\mathbf{L}_{t}$ is concave and $\mathbf{L}_{t}(0)=0$, we have $\mathbf{L}_{t}(\lambda x) \geq \lambda \mathbf{L}_{t}(x)$ for all $\lambda \in[0,1]$. Hence, $\lambda \mapsto \frac{\mathbf{L}_{t}(\lambda x)}{\lambda}$ is a non decreasing function. The function $D_{{ }_{+}} \mathbf{L}_{t}(0)$ is then well-defined and dominates $\mathbf{L}_{t}(\cdot)$. Moreover, for $\mu>0$ we have

$$
\lim _{\lambda \rightarrow 0} \frac{\mathbf{L}_{t}(\lambda \mu x)}{\lambda}=\mu \lim _{\lambda \mu \rightarrow 0} \frac{\mathbf{L}_{t}(\lambda \mu x)}{\lambda \mu}=\mu D_{x+} \mathbf{L}_{t}(0),
$$

i.e. $x \mapsto D_{x+} \mathbf{L}_{t}(0)$ is homogeneous of degree one. Now, consider $\mu, \eta>0$ and $\lambda>0$ small enough such that $\frac{\lambda}{\mu}+\frac{\lambda}{\eta} \leq 1$. Since $\mathbf{L}_{t}$ is concave and $\mathbf{L}_{t}(0)=0$, we get that

$$
\mathbf{L}_{t}(\lambda(x+y)) \geq \frac{\lambda}{\mu} \mathbf{L}_{t}(\mu x)+\frac{\lambda}{\eta} \mathbf{L}_{t}(\eta y)
$$

Therefore,

$$
D_{(x+y)+} \mathbf{L}_{t}(0)=\lim _{\lambda \rightarrow 0} \frac{\mathbf{L}_{t}(\lambda(x+y))}{\lambda} \geq \frac{\mathbf{L}_{t}(\mu x)}{\mu}+\frac{\mathbf{L}_{t}(\eta y)}{\eta} .
$$


Taking the limits as $\mu, \eta \rightarrow 0$, we conclude that $D{ }^{+} \mathbf{L}_{t}(0)$ is super-additive. Since $\widetilde{\mathbf{L}}_{t}$ is the smallest super-additive function dominating $\mathbf{L}_{t}$, we deduce that $\widetilde{\mathbf{L}}_{t}(\cdot) \leq D_{{ }_{+}} \mathbf{L}_{t}(0)$. Reciprocally, notice that by definition of $\widetilde{\mathbf{L}}_{t}$,

$$
\widetilde{\mathbf{L}}_{t}(x) \geq \sum_{i=1}^{n} \mathbf{L}_{t}\left(\frac{x}{n}\right)=n \mathbf{L}_{t}\left(\frac{x}{n}\right) .
$$

As $n$ tends to infinity, we get that $\widetilde{\mathbf{L}}_{t}(x) \geq D_{x+} \mathbf{L}_{t}(0)$ for all $x \in \mathbf{R}^{d}$. Therefore $\widetilde{\mathbf{L}}_{t}=D_{{ }_{+}} \mathbf{L}_{t}(0)$. By Proposition 2.3, the function $\widetilde{\mathbf{L}}_{t}$ satisfies (2.2) with $\widetilde{G}_{t}$ in place of $G_{t}$.

(ii) It is clear that $\overline{\mathbf{R}_{+} G_{t}}=: \widehat{G}_{t} \subseteq \widetilde{G}_{t}$. Reciprocally, since $\widehat{G}_{t}$ and $\widetilde{G}_{t}$ are both closed convex cones, it suffices to check that int $\widetilde{G}_{t} \subseteq \widehat{G}_{t}$, [29, Theorem 6.3]. Let $x \in \operatorname{int} \widetilde{G}_{t}$, it is immediate that $\widetilde{\mathbf{L}}_{t}(x)>0$ ( see [25, Proposition 2.6]). Moreover, since $\widetilde{\mathbf{L}}_{t}(x)=\lim _{\lambda \rightarrow 0+} \frac{\mathbf{L}_{t}(\lambda x)}{\lambda}$, there exists $\lambda>0$ small enough such that $\mathbf{L}_{t}(\lambda x)>0$, hence $\lambda x \in G_{t}$ and finally $x \in \widehat{G}_{t}$. We then conclude that $\widehat{G}_{t}=\widetilde{G}_{t}$.

\section{References}

[1] Almgren R. and Chriss N. Optimal execution of portfolio transactions. J. Risk, 2000, 3(2), 5-39.

[2] Astic F. and Touzi N. No arbitrage conditions and liquidity. Journal of Mathematical Economics, 2007, 43(6), 692-708.

[3] Bank P. and Baum D. Hedging and portfolio optimization in financial markets with a large trader. Mathematical Finance, 2004, 14(1), 1-18.

[4] Bouchard B, Loeper G. and Zou Y. Almost-sure hedging with permanent price impact, Preprint, 2015, arXiv:1503.05475.

[5] Brennan M.J. The optimal number of securities in a risky asset portfolio when there are fixed costs of transacting. Journal of Financial and Quantitative analysis, 1975, 10, 483-496.

[6] Cetin U., Jarrow R. and Protter P. Liquidity risk and arbitrage pricing theory. Finance and Stochastics, 2004, 8(3), 311-341.

[7] Cvitanić J. and Karatzas I. Hedging and portfolio optimization under transaction costs: a martingale approach. Mathematical Finance, 1996, 6(2), 133-165.

[8] Dalang E. C., Morton A. and Willinger W. Equivalent martingale measures and no-arbitrage in stochastic securities market models. Stochastics and Stochastic Reports, 1990, 29, 185-201. 
[9] Davis M. and Norman A. Portfolio selection with transaction costs. Math. Oper. Res., 1990, 15, 676-713.

[10] Delbaen F. and Schachermayer W. The mathematics of arbitrage. Springer, Berlin-Heidelberg-New York, 2006.

[11] Dolinsky Y. and Soner H.M. Duality and convergence for binomial markets with friction. Finance and Stochastics, 2013, 17(3), 447-475.

[12] Guasoni P., Lépinette E. and Rásonyi M. The fundamental theorem of asset pricing under transaction costs. Finance and Stochastics, 2012, 16(4), 741-777.

[13] Guasoni P., Rásonyi M. and Schachermayer, W. The fundamental theorem of asset pricing for continuous processes under small transaction costs. Annals of Finance, 2010, 6(2), 157-191.

[14] Hodges S. D. and Neuberger, A. Optimal replication of contingent claims under transaction costs, Review of Futures Markets, 1989, 8, 222-239.

[15] Jouini E. and Kallal H. Martingales and arbitrage in securities markets with transaction costs. J. Econ. Theory, 1995, 66, 178-97.

[16] Jouini E., Kallal H., and Napp C. Arbitrage and viability in securities markets with fixed trading costs. Journal of Mathematical Economics, 2001, 35, 197-221.

[17] Jouini E. and Napp C. Arbitrage with fixed costs and interest rate models. Journal of Financial and Quantitative Analysis, 2006, 41, 889913.

[18] Lépinette-Denis E. Arbitrage pricing under transaction costs: continuous time. Proceedings of the 2008 Daiwa International Workshop on Financial Engineering, Ed. World Scientific 2009, 91-106.

[19] Kabanov Y., Rásonyi M. and Stricker C. No-arbitrage criteria for financial markets with efficient friction. Finance and Stochastics, 2002, 6,371382 .

[20] Kabanov Y. On the FTAP of Kreps-Delbaen-Schachermayer. Statistics and Control of Stochastic Processes, Moscow, 1997,191-203.

[21] Kabanov Y. and Last G. Hedging under transaction costs in currency markets: a continuous-time model. Mathematical Finance, 2002, 12(1), 63-70.

[22] Kabanov Y. Hedging and liquidation under transaction costs in currency markets. Finance and Stochastics, 1999, 3(2), 237-248.

[23] Kabanov Y. and Safarian M. Markets with transaction costs. Mathematical Theory. Springer-Verlag, 2009. 
[24] Kabanov Y. and Lépinette E. Consistent price systems and arbitrage opportunities of the second kind in models with transaction costs. Finance and Stochastics, 2011, 16(1), 135-154.

[25] Lepinette E. and Tran T. General financial market model defined by a liquidation value process. To appear in Stochastics, 2015.

[26] Ma J., Song Q., Xu J. and Zhang J. Optimal portfolio selection under concave price impact. Applied Mathematics \& Optimization, 2013, 67(3),353-390.

[27] Oksendal B. and Sulem A. Optimal consumption and portfolio with both fixed and proportional transaction costs. SIAM Journal on Control and Optimization, 2002, 40(6), 1765-1790

[28] Penner I. and Pennanen T. Hedging of claims with physical delivery under convex transaction costs. SIAM Journal on Financial Mathematics, 2010, 1, 158-178.

[29] Rockafellar T. Convex analysis. Princeton University Press, Princeton, 1997.

[30] Shreve S.E. and Soner H.M. Optimal investment and consumption with transaction costs. Ann. Appl. Probab. 1994, 4(3), 609-692.

[31] Schachermayer W. The fundamental theorem of asset pricing under proportional transaction costs in finite discrete time. Mathematical Finance, 2004, 14(1), 19-48.

[32] Zakamouline V.I. European option pricing and hedging with both fixed and proportional transaction costs. Journal of Economic Dynamics and Control, 2006, 30(1), 1-25. 